Discrete Comput Geom 28:649-670 (2002)

DOI: $10.1007 /$ s00454-002-2897-y

\title{
Independence Numbers of Planar Contact Graphs
}

\author{
Konrad J. Swanepoel \\ Department of Mathematics, Applied Mathematics and Astronomy, \\ University of South Africa, PO Box 392, \\ Unisa 0003 , South Africa \\ swanekj@unisa.ac.za
}

\begin{abstract}
We show that for a large class of convex discs $C$ (including strictly convex discs), there exists an $\varepsilon=\varepsilon(C)>0$ such that the independence number of the contact graph of any packing of $n$ translates of $C$ in the plane is at least $\left(\frac{1}{4}+\varepsilon\right) n$. For $C$ a circle, we improve the lower bound of Csizmadia to $\frac{8}{31} n$.
\end{abstract}

\section{Introduction}

If we are given a set $P$ of $n$ points in the plane with minimum distance 1 , and we join two points whenever they are at distance 1 , we obtain the minimum distance graph $G(P)$ of $P$. Given any graph $G$, a subset of its vertices is independent if no two vertices in the set are adjacent in $G$. The independence number of $G$ is the largest size $\alpha(G)$ of an independent subset of $G$. Let $F(n)$ denote the smallest possible independence number that a minimum distance graph on $n$ points in the plane can have:

$$
F(n)=\min _{|P|=n} \alpha(G(P))
$$

In 1983 Erdôs asked for estimates of $F(n)$. Chung, Graham and Pach found an upper bound of $\frac{6}{19} n$ (see [3]), which was improved to $\frac{5}{16} n$ by Pach and Tóth [5]. By observing that such a graph is planar, hence four-colourable, Pollack [6] obtained the lower bound of $n / 4$, which was improved by Csizmadia [2] to $\frac{9}{35} n$. Our first result is a slight improvement.

Theorem 1. For any set of $n$ points in the plane with minimum distance 1 , there exists a subset of at least $\frac{8}{31} n$ points such that the distance between any two of these points is more than 1 . 


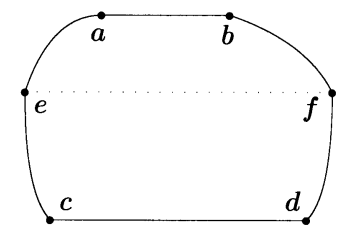

Fig. 1. A paralleloid: $\boldsymbol{a b}+\boldsymbol{c d}>\boldsymbol{e f}$.

By drawing for each $\boldsymbol{p} \in P$ a circle of diameter 1 with centre $\boldsymbol{p}$, we obtain a packing of congruent circles, and the minimum distance graph of $P$ can now be considered to be the contact graph of the packing. The question of Erdôs can be generalized to contact graphs of translates of any convex disc $C$. Let $F_{C}(n)$ denote the smallest possible independence number that the contact graph of a packing of $n$ translates of $C$ can have. It is easily seen that if $C$ is a parallelogram, then $F_{C}(n)=\lceil n / 4\rceil$. If $C$ is not a parallelogram, then contact graphs are again planar, and we have the lower bound $F_{C}(n) \geq n / 4$. We note that the four-colourability of minimum distance graphs does not need the four-colour theorem, since it is easily seen that there always exists a vertex of degree at most 3 , hence the graph can be coloured in a greedy manner.

We improve this lower bound of $n / 4$ for a large class of convex discs, including strictly convex ones. We call a convex disc $C$ a paralleloid if there exist two parallel supporting lines to $C$, intersecting $C$ in two line segments $\boldsymbol{a} \boldsymbol{b}$ and $\boldsymbol{c d}$ such that the sum of the lengths of $\boldsymbol{a b}$ and $\boldsymbol{c d}$ is strictly larger than the length of the intersection of $C$ and any line parallel to the two supporting lines. See Fig. 1.

Theorem 2. If $C$ is not a paralleloid, then there exists a constant $c>\frac{1}{4}$ depending on $C$, such that $F_{C}(n) \geq c n$.

We note that the $\frac{5}{16} n$ upper bound of Pach and Tóth for the case of a circle easily generalizes to any $C$. In the next section we indicate the connection between contact graphs and minimum distance graphs in a normed plane, and introduce an angular measure due to Brass [1] in normed planes. In Section 3 we prepare the way for the proofs of Theorem 1 in Section 4 and Theorem 2 in Section 5.

\section{Contact Graphs and Minimum Distance Graphs}

Let $C$ be a convex disc, i.e., a compact convex body in the plane $\mathbf{R}^{2}$. A translate of $C$ is a disc of the form $C+\boldsymbol{x}:=\{\boldsymbol{c}+\boldsymbol{x}: \boldsymbol{c} \in C\}$ for some $\boldsymbol{x} \in \mathbf{R}^{2}$. Two translates touch (overlap) if they have only boundary points in common (if they have interior points in common). A collection $\mathcal{C}=\left\{C_{1}, \ldots, C_{n}\right\}$ of translates of $C$ is a packing if no two translates overlap. The contact graph $G(\mathcal{C})$ of $\mathcal{C}$ has the translates as vertices, and touching pairs of translates as edges.

The difference body of $C$ is $C-C:=\left\{\boldsymbol{c}_{1}-\boldsymbol{c}_{2}: \boldsymbol{c}_{1}, \boldsymbol{c}_{2} \in C\right\}$, which is well known to be convex and centrally symmetric. It is also well known that two translates $C+x_{1}$ and $C+\boldsymbol{x}_{2}$ touch, overlap or are disjoint iff $\frac{1}{2}(C-C)+\boldsymbol{x}_{1}$ and $\frac{1}{2}(C-C)+\boldsymbol{x}_{2}$ touch, 
overlap or are disjoint, respectively. Thus the graph structure stays the same if we replace a convex disc by its difference body. It is well known that $C$ is a parallelogram iff its difference body is a parallelogram. It is also readily seen that $C$ is a paralleloid iff its difference body is a paralleloid.

\section{Normed Planes}

The unit ball of any normed plane is a centrally symmetric convex disc. Conversely, given any convex disc $C$ symmetric around the origin $\boldsymbol{o}$, there exists a norm turning the plane into a two-dimensional normed space (or Minkowski plane), denoted by $M(C)$, such that $C$ is the unit ball of the norm:

$$
\|x\|=\|x\|_{C}:=\inf \left\{\lambda: \lambda>0, \lambda^{-1} x \in C\right\} .
$$

We denote the distance between $\boldsymbol{x}_{1}$ and $\boldsymbol{x}_{2}$ in the norm by

$$
x_{1} x_{2}:=\left\|x_{1}-x_{2}\right\|
$$

It is clear that $C+\boldsymbol{x}_{1}$ and $C+\boldsymbol{x}_{2}$ touch, overlap or are disjoint iff $\boldsymbol{x}_{1} \boldsymbol{x}_{2}$ equals, is less than or is greater than 2, respectively. It follows that the contact graph of the packing $\left\{C+\boldsymbol{x}_{i}: i=1, \ldots, n\right\}$ is exactly the minimum distance graph of the points $\left\{\boldsymbol{x}_{1}, \ldots, \boldsymbol{x}_{n}\right\}$. In our proofs we therefore consider only minimum distance graphs.

A segment contained in bd $C$ of length (in the norm determined by $C$ ) more than 1 is called a long segment. We need the following easily proven characterizations of parallelograms and paralleloids.

Proposition 1. Let $M(C)$ be a normed plane with unit ball $C$.

1. There exists a long segment on the boundary of $C$ iff $C$ is a paralleloid.

2. A long segment has length at most 2 , with equality iff $C$ is a parallelogram.

By the Mazur-Ulam theorem [8] two centrally symmetric convex discs $C_{1}$ and $C_{2}$ are affinely equivalent iff the normed planes $M\left(C_{1}\right)$ and $M\left(C_{2}\right)$ are isometric. We therefore call any normed plane with a parallelogram as the unit ball the rectilinear plane. It is also clear that if two convex discs are affinely equivalent, then they determine the same collection of contact graphs.

We note the following well-known properties of minimum distance graphs in nonrectilinear normed planes (see [1] and [4]).

Proposition 2. If $M(C)$ is nonrectilinear, then any minimum distance graph in $M(C)$ is planar, and the maximum degree is at most 6.

\section{Angular Measures}

An important tool that we use is an angular measure defined in any normed plane except the rectilinear plane, due to Brass [1]. Let bd $C$ be the unit circle of the normed plane 
$M(C)$. An angular measure on $M(C)$ is a measure $\mu$ on bd $C$ satisfying

$$
\begin{aligned}
\mu(\mathrm{bd} C) & =2 \pi, \\
\mu(A) & =\mu(-A) \text { for all measurable } A \subseteq \operatorname{bd} C, \\
\mu(\{\boldsymbol{p}\}) & =0 \text { for all } \boldsymbol{p} \in \mathrm{bd} C .
\end{aligned}
$$

We then measure an angle in the obvious translation invariant way. By $\varangle \boldsymbol{a b c}$ we denote the angle corresponding to the positive (counterclockwise) rotation of the ray $\overrightarrow{\boldsymbol{b} \boldsymbol{a}}$ to $\overrightarrow{\boldsymbol{b} \boldsymbol{c}}$. In a simple closed polygon $\boldsymbol{p}_{1} \boldsymbol{p}_{2} \cdots \boldsymbol{p}_{n}$ we denote the interior angle at $\boldsymbol{p}_{i}$ by $\varangle \boldsymbol{p}_{i}$. We always number consecutive vertices in the positive (counterclockwise) sense, thus $\varangle \boldsymbol{p}_{i}$ is exactly $\varangle \boldsymbol{p}_{i+1} \boldsymbol{p}_{i} \boldsymbol{p}_{i-1}$. An angular measure is proper if $\mu(A)>0$ for any nontrivial arc $A$ of bd $C$. An angular measure is a Brass measure if equilateral triangles in $M(C)$ are equiangular in the measure.

Proposition 3 [1]. The normed plane $M(C)$ admits a Brass measure iff $C$ is not a parallelogram. There exists a proper Brass measure iff $C$ is not a paralleloid.

The following properties of Brass measures will be used repeatedly. Properties 1 and 2 are used extensively in [1]. It is rather surprising that property 3, already not entirely trivial in the Euclidean plane, still holds in this generality.

Proposition 4. Let $\mu$ be any Brass measure in any nonrectilinear normed plane.

1. The angle sum of a simple closed $n$-gon is $\pi(n-2)$.

2. If $\boldsymbol{p q}=\boldsymbol{q r} \leq \boldsymbol{p r}$ in $\triangle \boldsymbol{p q r}$, then $\mu(\varangle \boldsymbol{q}) \geq \pi / 3$. Equality implies that the triangle is equilateral whenever $\mu$ is proper.

3. Let abcd be a simple quadrilateral with $\boldsymbol{a b}=\boldsymbol{b c}=\boldsymbol{c d} \leq \boldsymbol{a d}, \boldsymbol{a c}, \boldsymbol{b d}$. Then $\mu(\varangle \boldsymbol{b})+\mu(\varangle \boldsymbol{c}) \geq \pi$.

Properties 2 and 3 have the following implications for the faces of minimum distance graphs:

Corollary 3. In a minimum distance graph in any nonrectilinear normed plane with Brass measure $\mu$,

1. any interior angle of a face is at least $\pi / 3$, and for proper $\mu$ equality is possible only if the face is a triangle, and

2. the sum of any two consecutive interior angles of a nontriangularface is at least $\pi$.

Proof of Proposition 4. Property 1 is proved exactly as in the Euclidean case: a simple closed $n$-gon can be triangulated into $n-2$ triangles, while the classical Euclidean proof that the angle sum of a triangle is $\pi$ carries over verbatim. Note that this result still holds even if the measure is not a Brass measure.

Property 2 is immediate from the properties of a Brass measure.

Property 3: By scaling we may assume that $\boldsymbol{a b}=1$. Suppose $\boldsymbol{a b} \boldsymbol{c d}$ is not convex. If $\boldsymbol{b}$ is in $\triangle \boldsymbol{a c d}$, then $\mu(\varangle \boldsymbol{b}) \geq \pi$. Similarly, if $\boldsymbol{c}$ is in $\triangle \boldsymbol{a b d}$, then $\mu(\varangle \boldsymbol{c}) \geq \pi$. If $\boldsymbol{a}$ is in $\triangle \boldsymbol{b c d}$ 


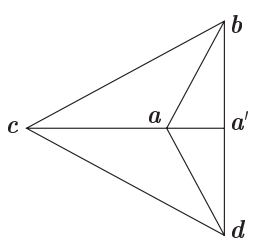

(a)

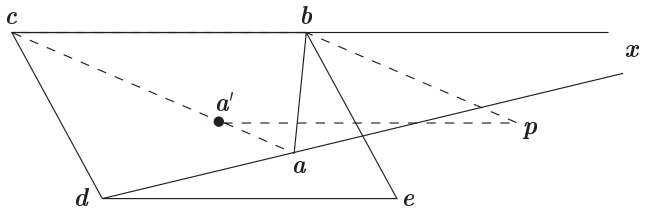

(b)

Fig. 2. Proof of Proposition 4.

(the case $\boldsymbol{d}$ in $\triangle \boldsymbol{a b} \boldsymbol{c}$ is similar), let $\boldsymbol{a}^{\prime}$ be the point at which the ray $\overrightarrow{\boldsymbol{c a}}$ intersects $\boldsymbol{b} \boldsymbol{d}$. See Fig. 2(a). We have $\boldsymbol{a}^{\prime}=\lambda \boldsymbol{b}+(1-\lambda) \boldsymbol{d}$ for some $0 \leq \lambda \leq 1$, hence

$$
\begin{aligned}
1 & \leq \boldsymbol{c a} \leq \boldsymbol{c} \boldsymbol{a}^{\prime} \\
& =\left\|\boldsymbol{c}-\boldsymbol{a}^{\prime}\right\|=\|\lambda(\boldsymbol{c}-\boldsymbol{b})+(1-\lambda)(\boldsymbol{c}-\boldsymbol{d})\| \\
& \leq \lambda \boldsymbol{b c}+(1-\lambda) \boldsymbol{c d}=1 .
\end{aligned}
$$

Thus $\boldsymbol{c} \boldsymbol{a}=\boldsymbol{c} \boldsymbol{a}^{\prime}$ and $\boldsymbol{a}=\boldsymbol{a}^{\prime}$. Thus $\boldsymbol{b}, \boldsymbol{a}$ and $\boldsymbol{d}$ are three collinear points on the boundary of the unit ball $C+\boldsymbol{c}$. Then $\boldsymbol{b} \boldsymbol{d}$ is a segment of length 2 (in the norm) on the boundary of $C+c$. By Proposition 1 it follows that $C$ is a parallelogram, a contradiction. It follows that $\boldsymbol{a b c d}$ is convex.

If $\overleftrightarrow{\boldsymbol{a d}}$ and $\overleftrightarrow{\boldsymbol{b c}}$ are parallel, then $\boldsymbol{a b c d}$ is a parallelogram, and $\mu(\varangle \boldsymbol{b})+\mu(\varangle \boldsymbol{c})=\pi$ is immediate. Thus assume that the lines $\overleftrightarrow{\boldsymbol{a d}}$ and $\overleftrightarrow{\boldsymbol{b c}}$ intersect in a point $\boldsymbol{x}$. Without loss of generality $\boldsymbol{x}$ is on the rays $\overrightarrow{\boldsymbol{c} b}$ and $\overrightarrow{\boldsymbol{d a}}$ (otherwise interchange $\boldsymbol{a} \leftrightarrow \boldsymbol{d}, \boldsymbol{b} \leftrightarrow \boldsymbol{c}$ ). See Fig. 2(b). Let $\boldsymbol{e}$ be the point such that $\boldsymbol{b c d e}$ is a parallelogram (i.e., $\boldsymbol{e}=\boldsymbol{b}+\boldsymbol{d}-\boldsymbol{c}$ ). If $\boldsymbol{a}$ is outside $\boldsymbol{b c d e}$, then

$$
\begin{aligned}
\mu(\varangle \boldsymbol{b})+\mu(\varangle \boldsymbol{c}) & =\mu(\varangle \boldsymbol{e b} \boldsymbol{a})+\mu(\varangle \boldsymbol{c} \boldsymbol{b} \boldsymbol{e})+\mu(\varangle \boldsymbol{c}) \\
& =\mu(\varangle \boldsymbol{e b} \boldsymbol{a})+\pi \geq \pi .
\end{aligned}
$$

Similarly, if $\boldsymbol{a}$ is on $\boldsymbol{b} \boldsymbol{e}$, then $\mu(\varangle \boldsymbol{b})+\mu(\varangle \boldsymbol{c})=\pi$. If $\boldsymbol{a}$ is on $\boldsymbol{d e}$, then, since $\boldsymbol{a d}=\boldsymbol{d} \boldsymbol{e}=1$, we have $\boldsymbol{a}=\boldsymbol{e}$, a case already dealt with.

In the remaining case, $\boldsymbol{a}$ is in the interior of $\boldsymbol{b c d e}$. Since $\boldsymbol{b c}=\boldsymbol{b} \boldsymbol{a}=\boldsymbol{b} \boldsymbol{e}=1$, it follows from the convexity of the unit ball $C+\boldsymbol{b}$ that $\boldsymbol{a}$ is not in the interior of $\triangle \boldsymbol{b c e}$. Similarly, since $1=\boldsymbol{d} \boldsymbol{c}=\boldsymbol{d e} \leq \boldsymbol{d a}, \boldsymbol{a}$ is not in the interior of $\triangle \boldsymbol{c d e}$. Thus $\boldsymbol{a}$ is on $\boldsymbol{c e}, \boldsymbol{d a}=1$, and $\boldsymbol{c} \boldsymbol{e}$ is a segment contained in the boundaries of $\boldsymbol{C}+\boldsymbol{b}$ and $\boldsymbol{C}+\boldsymbol{d}$. Let $\boldsymbol{a}^{\prime}$ be the point on segment $\boldsymbol{c} \boldsymbol{a}$ such that $\boldsymbol{c} \boldsymbol{a}^{\prime}=1$. Then $\triangle \boldsymbol{a}^{\prime} \boldsymbol{c d}$ is equilateral. If we let $\boldsymbol{p}=\boldsymbol{a}^{\prime}+\boldsymbol{b}-\boldsymbol{c}$, then $\triangle \boldsymbol{p} \boldsymbol{b} \boldsymbol{e}$ is $\triangle \boldsymbol{a}^{\prime} \boldsymbol{c} \boldsymbol{d}$ translated by $\boldsymbol{b}-\boldsymbol{c}$, hence also equilateral, and $\mu(\varangle \boldsymbol{e b p})=\pi / 3$. Also, since segment $\boldsymbol{c} \boldsymbol{e}$ is on the boundary of $\boldsymbol{C}+\boldsymbol{b}$, it follows that $\boldsymbol{a}^{\prime} \boldsymbol{e}$ is on the boundary of $C+\boldsymbol{p}$, which is $C+\boldsymbol{b}$ translated by $\boldsymbol{a}^{\prime}-\boldsymbol{c}$. Thus $\boldsymbol{p} \boldsymbol{a}=1$. Since $\boldsymbol{b} \boldsymbol{a}=1$ and $\boldsymbol{b} \boldsymbol{p}=\boldsymbol{c} \boldsymbol{a}^{\prime}=1$ we have that $\triangle \boldsymbol{a b p}$ is equilateral and $\mu(\varangle \boldsymbol{a b p})=\pi / 3$. Thus $\mu(\varangle \boldsymbol{a b e})=0$, and

$$
\begin{aligned}
\mu(\varangle \boldsymbol{b})+\mu(\varangle \boldsymbol{c}) & =\mu(\varangle \boldsymbol{b})+\mu(\varangle \boldsymbol{e b} \boldsymbol{x}) \\
& =\mu(\varangle \boldsymbol{c} \boldsymbol{b} \boldsymbol{e})+\mu(\varangle \boldsymbol{e b} \boldsymbol{x}) \\
& =\pi .
\end{aligned}
$$


The following corollary of Proposition 4, property 3, will be used.

Proposition 5. Let $M(C)$ be a nonrectilinear normed plane.

(1) A four-cycle in a minimum distance graph is convex.

(2) The complete bipartite graph $K_{2,3}$ cannot occur as a subgraph of a minimum distance graph.

Proof. (1) By inspecting the proof of Proposition 4, property 3, it is seen that it is sufficient to consider the case where $\boldsymbol{b}$ is in $\triangle \boldsymbol{a c d}$, and derive a contradiction. Let $\boldsymbol{b}^{\prime}$ and $\boldsymbol{d}^{\prime}$ be such that $\boldsymbol{a} \boldsymbol{b}^{\prime} \boldsymbol{c} \boldsymbol{b}$ and $\boldsymbol{a d} \boldsymbol{d}^{\prime} \boldsymbol{c d}$ are parallelograms, i.e., $\boldsymbol{b}^{\prime}=\boldsymbol{a}-\boldsymbol{b}+\boldsymbol{c}$ and $\boldsymbol{d}^{\prime}=\boldsymbol{a}-\boldsymbol{d}+\boldsymbol{c}$. Then $\boldsymbol{b}, \boldsymbol{b}^{\prime}, \boldsymbol{d}, \boldsymbol{d}^{\prime}$ are on the boundary of the unit ball $C+\boldsymbol{a}$. Since the unit ball is convex and $\boldsymbol{b} \boldsymbol{d}$ and $\boldsymbol{b}^{\prime} \boldsymbol{d}^{\prime}$ are two parallel segments, it follows that they are on the same line, and $\boldsymbol{d} \boldsymbol{d}^{\prime}$ is a segment on the boundary of $C+\boldsymbol{a}$, of length at least $\boldsymbol{b d}+\boldsymbol{b}^{\prime} \boldsymbol{d}^{\prime} \geq 2 \boldsymbol{a b}$. By Proposition $1 C$ is a parallelogram, a contradiction.

(2) It is impossible to draw $K_{2,3}$ with classes $\{\boldsymbol{a}, \boldsymbol{b}\}$ and $\{\boldsymbol{p}, \boldsymbol{q}, \boldsymbol{r}\}$ such that the quadrilaterals $\boldsymbol{a p b q}, \boldsymbol{a p b r}$ and $\boldsymbol{a q b r}$ are all convex.

Note that $K_{2,3}$ can occur as a unit distance graph in certain normed planes, in fact exactly when the unit ball is a paralleloid [1]. Also, $K_{2,3}$ can occur as a (noninduced) subgraph of a minimum distance graph in the rectilinear plane.

\section{Proofs of Theorems 1 and 2: Generalities}

We prove Theorems 1 and 2 by induction on the number of points $n$. A large part of the proof holds for any nonrectilinear plane, and, thanks to the Brass measure, is not made any simpler by assuming that we are working in the Euclidean plane. This general part of the proof is contained in this section. It essentially consists of showing that a minimal counterexample to Theorem 2 contains a specific type of a "broken lattice" configuration whose size depends on $c>\frac{1}{4}$. In the next section we show that such a configuration cannot exist in the Euclidean plane if $c=\frac{8}{31}$, thus proving Theorem 1. In Section 5 we show that for each nonparalleloid $C$ there is a sufficiently small $c>0$ such that the above-mentioned "broken lattice" cannot exist in $M(C)$, thus proving Theorem 2. In order to extend Theorem 2 to other nonrectilinear normed planes, we would only have to prove the nonexistence of a sufficiently large broken lattice configuration in those planes. (See Theorem 4 at the end of this section.)

Everything in this section occurs in a nonrectilinear normed plane $M(C)$ for which an arbitrary Brass measure has been chosen. We fix an integer $m \geq 5$ to be determined later (depending only on the norm) and let $c=m /(4 m-1)$. We now consider a smallest counterexample to $F_{C}(n) \geq c n$, i.e., we fix a set $P$ of $n$ points in $M(C)$ with minimum distance graph $G$ such that

$$
\alpha(G)<c n
$$

and, secondly,

$$
\text { for any } n^{\prime}<n \quad \text { we have } \quad F_{C}\left(n^{\prime}\right) \geq c n^{\prime} \text {. }
$$


We remark that considering a smallest counterexample is not essential in this proof, which could have been presented as an algorithmic proof for finding a large independent subset of any given $n$ points, but at the cost of complicating the exposition. We now use assumptions (1) and (2) to prove a sequence of lemmas describing properties of the minimal counterexample, culminating in the necessity of containing a "broken lattice" configuration, the description of which will be made precise in the course of the deduction. The first property following from (1) and (2) is an observation of Csizmadia [2].

Lemma 1. Any independent subset $S \subseteq G$ of at most $k$ points $(k \leq m)$ has at least $3 k$ neighbours in $G-S$.

Proof. Suppose that $G$ has an independent subset $S$ of $k$ points with less than $3 k$ neighbours in $G-S$. Delete the neighbours of $S$ from $G-S$ to form a smaller graph $G^{\prime}$ with $n^{\prime}$ points, $n^{\prime} \geq n-(4 k-1)$. By (2) $G^{\prime}$ has an independent subset $S^{\prime}$ of size at least $(m /(4 m-1)) n^{\prime}$. (Note that if $G^{\prime}$ does not have any edges, then it is not a minimum distance graph and (2) does not apply, but then there is an independent subset of size $n^{\prime}$.) Then $S \cup S^{\prime}$ is an independent subset of $G$ of size at least

$$
\begin{aligned}
c n^{\prime}+k & \geq c(n-4 k+1)+k \\
& =c n+\frac{m-k}{4 m-1} \geq c n,
\end{aligned}
$$

which contradicts (1).

Applying Lemma 1 to a set $S$ consisting of a single point we obtain

Lemma 2. $G$ has minimum degree at least 3 .

Lemma 3. $G$ is 2-connected.

Proof. If $G$ is not connected we apply (2) to find an independent subset of each connected component, the union of which gives an independent subset of $G$ that contradicts (1).

Suppose then that $G$ has a cut vertex $\boldsymbol{x}$. Let $G_{1}$ and $G_{2}$ be the two components of $G-\boldsymbol{x}$, and let $G_{1}^{\prime}=G-G_{2}$ and $G_{2}^{\prime}=G-G_{1}$. If $\alpha\left(G_{1}^{\prime}\right)=\alpha\left(G_{1}\right)$, then $G_{1}$ (hence also $\left.G_{1}^{\prime}\right)$ has an independent subset of size $\alpha\left(G_{1}^{\prime}\right)$ not containing $\boldsymbol{x}$. Applying (2) to both $G_{1}^{\prime}$ and $G_{2}$ we then obtain an independent subset of $G$ contradicting (1). Thus $\alpha\left(G_{1}^{\prime}\right)=\alpha\left(G_{1}\right)+1$, hence any independent subset $S_{1}$ of $G_{1}^{\prime}$ of size $\alpha\left(G_{1}^{\prime}\right)$ contains $\boldsymbol{x}$. Then $S_{1} \backslash\{\boldsymbol{x}\}$ is an independent subset of $G_{1}$ not containing any neighbour of $\boldsymbol{x}$, and we may apply (2) to $G_{1}$ and $G_{2}^{\prime}$ to obtain an independent subset of $G$ contradicting (1).

Thus $G$ does not have any cut vertices.

Since $G$ is 2-connected and planar (Proposition 2) it has a simple boundary polygon. We label the vertices of this polygon in the positive (counterclockwise) orientation by $\boldsymbol{p}_{1}, \boldsymbol{p}_{2}, \ldots, \boldsymbol{p}_{t}$. We also let $\boldsymbol{p}_{-1}=\boldsymbol{p}_{t-1}, \boldsymbol{p}_{0}=\boldsymbol{p}_{t}$ and $\boldsymbol{p}_{t+1}=\boldsymbol{p}_{1}$. 
We now analyze this polygon and its immediate neighbourhood. This requires the introduction of the following notation and terminology. We distinguish between two types of boundary edge: $\boldsymbol{p}_{i} \boldsymbol{p}_{i+1}$ is a triangle boundary edge if $\boldsymbol{p}_{i}$ and $\boldsymbol{p}_{i+1}$ have a common neighbour, otherwise $\boldsymbol{p}_{i} \boldsymbol{p}_{i+1}$ is a nontriangle boundary edge. Note that the endpoints of a triangle boundary edge $\boldsymbol{p}_{i} \boldsymbol{p}_{i+1}$ can have only one common neighbour, which we denote $\boldsymbol{q}_{i}$. Indeed, if $\boldsymbol{p}_{i}$ and $\boldsymbol{p}_{i+1}$ have $\boldsymbol{q}$ and $\boldsymbol{q}^{\prime}$ as common neighbours with $\boldsymbol{q}$ inside the equilateral $\triangle \boldsymbol{p}_{i} \boldsymbol{p}_{i+1} \boldsymbol{q}^{\prime}$, then $\boldsymbol{p}_{i} \boldsymbol{q} \boldsymbol{p}_{i+1} \boldsymbol{q}^{\prime}$ is a nonconvex quadrilateral, contradicting Proposition 5.

Let $b$ be the number of nontriangle edges. Let $d_{i}$ be the number of boundary vertices of degree $i$ in $G(i=3,4,5,6)$. We remark without proof that in the Euclidean case boundary points cannot have degree 6 , but there exist normed planes (in fact, exactly when the unit ball is a paralleloid; see [7]) in which degree 6 boundary points are possible. However, taking degree 6 boundary points into account does not offer any difficulties at all, so this remark is not needed for the proof. We call the nontriangle edges as well as the boundary vertices of degree 5 and 6 negative elements. There are $N=b+d_{5}+d_{6}$ negative elements. We now find a lower estimate on the angle sum of the boundary polygon. Let $\theta_{i}=\mu\left(\boldsymbol{p}_{i}\right)$ be the Brass measure of the interior angle at vertex $\boldsymbol{p}_{i}$. We call $\tau_{i}=\theta_{i}-\pi$ the turn at $\boldsymbol{p}_{i}$ (measured in the negative sense as we go round the boundary in a counterclockwise direction). By Proposition 4, property 2, each boundary point of degree $i$ contributes at least $\pi(i-1) / 3$ to the angle sum, and by Proposition 4, property 3 , each nontriangle edge contributes at least $\pi$, hence at least $\pi / 3$ above that already accounted for. We call a maximal sequence of $k$ consecutive degree 4 boundary vertices $\boldsymbol{p}_{i+1}, \ldots, \boldsymbol{p}_{i+k}$ with only triangle edges between consecutive vertices an arc of size $k$ if one of the following holds:

1. $\operatorname{deg}\left(p_{i}\right)=3, \operatorname{deg}\left(p_{i-1}\right)=4$, and $\boldsymbol{p}_{i} \boldsymbol{p}_{i-1}$ and $\boldsymbol{p}_{i} \boldsymbol{p}_{i+1}$ are triangle edges,

2. $\operatorname{deg}\left(p_{i}\right)=\operatorname{deg}\left(p_{i-1}\right)=3$, and $\boldsymbol{p}_{i} \boldsymbol{p}_{i+1}$ is a triangle edge,

3. $\operatorname{deg}\left(p_{i+k+1}\right)=3, \operatorname{deg}\left(p_{i+k+2}\right)=4$, and $\boldsymbol{p}_{i+k} \boldsymbol{p}_{i+k+1}$ and $\boldsymbol{p}_{i+k+1} \boldsymbol{p}_{i+k+2}$ are triangle edges,

4. $\operatorname{deg}\left(p_{i+k+1}\right)=\operatorname{deg}\left(p_{i+k+2}\right)=3$, and $\boldsymbol{p}_{i+k} \boldsymbol{p}_{i+k+1}$ is a triangle edge.

See Fig. 3. Let $A$ be the number of arcs of size at least $2 m-3$. Note that in an arc of size $k$ we have $\tau_{i+1}, \ldots, \tau_{i+k} \geq 0$ since each vertex has degree 4 , hence has interior angle at least $\pi$ by Proposition 4 , property 2 . We call an arc $\boldsymbol{p}_{i+1}, \ldots, \boldsymbol{p}_{i+k}$ concave if the total turn $\tau_{i+1}+\cdots+\tau_{i+k} \geq \pi / 3$, i.e., if these vertices contribute a further $\pi / 3$ to the angle sum above that already accounted for. Let $B$ be the number of concave arcs of size at least $2 m-3$. By the previous discussion and Proposition 4, property 1 , the angle sum of the boundary polygon is

$$
\pi\left(\sum_{i=3}^{6} d_{i}-2\right)=\sum_{i=1}^{t} \theta_{i} \geq \sum_{i=3}^{6} \frac{\pi}{3}(i-1) d_{i}+\frac{\pi}{3}(b+B),
$$

and it follows that

$$
d_{3} \geq d_{5}+2 d_{6}+b+B+6 \geq N+B+6 .
$$




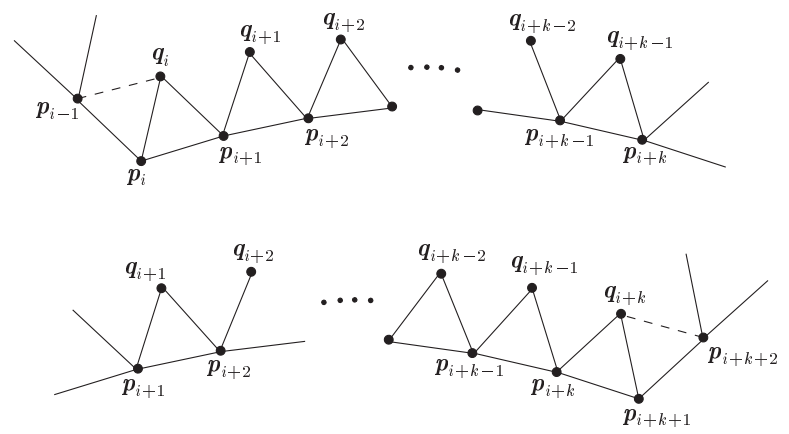

Fig. 3. Arcs of size $k$.

The above argument applied to any simple closed polygon in $G$ gives the following statement that will be needed in future:

Lemma 4. Let $G^{\prime}$ be the subgraph of $G$ induced by the boundary and interior of some simple closed polygon $Q$ in $G$. Let $D_{i}$ be the number of vertices of degree i in $G^{\prime}$ on the boundary of $Q$. Then $2 D_{2}+D_{3} \geq 6$.

The "broken lattice" configuration that we seek is the following.

Lemma 5. There is a nonconcave arc of size at least $2 m-3$.

After the proof of Lemma 5 we study the properties of such an arc. It will then become clear why the description "broken lattice" is appropriate.

Proof of Lemma 5. We have to show that $A>B$, so in the light of (3) it is more than sufficient to prove that

$$
N+A \geq d_{3}
$$

Let $\boldsymbol{p}_{i(1)}, \ldots, \boldsymbol{p}_{i\left(d_{3}\right)}$ be all the boundary vertices of degree 3 with $1 \leq i(1)<\cdots<$ $i\left(d_{3}\right) \leq t$. We let $i\left(d_{3}+1\right)=i(1)$. Inequality (4) is immediate if there is a negative element or an arc of size at least $2 m-3$ between any two successive degree 3 vertices. Thus assume without loss of generality that there is no negative element nor an arc of size at least $2 m-3$ between the first two degree 3 vertices $\boldsymbol{p}_{i(1)}$ and $\boldsymbol{p}_{i(2)}$. We let $\boldsymbol{p}_{i\left(d_{3}+1\right)}=\boldsymbol{p}_{i(1)}$. We first state and prove Lemmas 6 and 7 before concluding the proof of Lemma 5.

Lemma 6. For any $j=1, \ldots, d_{3}$, if there is no arc of size at least $2 m-3$ between $\boldsymbol{p}_{i(j)}$ and $\boldsymbol{p}_{i(j+1)}$, and also no negative element between $\boldsymbol{p}_{i(j)}$ and $\boldsymbol{p}_{i(j+1)}$ with the single exception of either the vertex $\boldsymbol{p}_{i(j)+1}$ or the edge $\boldsymbol{p}_{i(j)} \boldsymbol{p}_{i(j)+1}$, then $\boldsymbol{p}_{i(j+1)+1}$ or $\boldsymbol{p}_{i(j+1)} \boldsymbol{p}_{i(j+1)+1}$ is a negative element (perhaps both). 
Proof. We assume that $\operatorname{deg}\left(\boldsymbol{p}_{i(j+1)+1}\right) \leq 4$ and that $\boldsymbol{p}_{i(j+1)} \boldsymbol{p}_{i(j+1)+1}$ is a triangle edge and aim for a contradiction. By definition there is an arc of size at least $i(j+1)-i(j)-2$ between $\boldsymbol{p}_{i(j)}$ and $\boldsymbol{p}_{i(j+1)}$. By our assumption then $i(j+1)-i(j)-2<2 m-3$. Consider the vertices $\boldsymbol{p}_{i(j)+2 h}, h=0, \ldots,\lceil(i(j+1)-i(j)) / 2\rceil$. There are $k:=\lceil(i(j+1)-$ $i(j)) / 2\rceil+1$ of them, with $k \leq m$, and together they have at most $3 k-1$ neighbours (there are two cases to check depending on the parity of $i(j+1)-i(j))$. We now show that they are all independent, which together with Lemma 1 gives the required contradiction. The only cases where two of these points could possibly be identical are $\boldsymbol{p}_{i(j)}=\boldsymbol{p}_{i(j+1)}$ (if $i(j+1)-i(j)$ is even) or $\boldsymbol{p}_{i(j)}=\boldsymbol{p}_{i(j+1)+1}$ (if $i(j+1)-i(j)$ is odd). However, then there are only one or two vertices of degree 3 on the boundary, contradicting Lemma 4. Suppose next that $\boldsymbol{p}_{i(j)+2 a}$ and $\boldsymbol{p}_{i(j)+2 b}$ are adjacent for some $0 \leq a<b \leq\lceil(i(j+1)-i(j)) / 2\rceil$. Consider the subgraph $G^{\prime}$ of $G$ induced by the polygon $\boldsymbol{p}_{i(j)+2 a} \boldsymbol{p}_{i(j)+2 a+1} \cdots \boldsymbol{p}_{i(j)+2 b}$ and its interior. The only vertices of this polygon that could possibly have degree 3 in $G^{\prime}$ are $\boldsymbol{p}_{i(j)+2 a}, \boldsymbol{p}_{i(j)+2 b}, \boldsymbol{p}_{i(j)}, \boldsymbol{p}_{i(j+1)}$ and $\boldsymbol{p}_{i(j+1)+1}$ (with none of degree 2). This again contradicts Lemma 4.

Let $N_{j}$ be the number of negative elements and let $A_{j}$ be the number of arcs of size at least $2 m-3$ between $\boldsymbol{p}_{i(1)}$ and $\boldsymbol{p}_{i(j+1)}$ for $j=1, \ldots, d_{3}$. Thus $N_{d_{3}}=N$ and $A_{d_{3}}=A$. We now show by induction on $j$ that

Lemma 7. For each $j=1, \ldots, d_{3}$ we have $N_{j}+A_{j} \geq j-1$, and, furthermore, if $N_{j}+A_{j}=j-1$, then $\boldsymbol{p}_{i(j+1)+1}$ or $\boldsymbol{p}_{i(j+1)} \boldsymbol{p}_{i(j+1)+1}$ is a negative element (perhaps both).

Proof. The lemma already holds for $j=1$, since by our choice of $\boldsymbol{p}_{i(1)}$ and $\boldsymbol{p}_{i(2)}$, $N_{1}=0$, while if $A_{1}=0$, then Lemma 6 implies that $\boldsymbol{p}_{i(2)+1}$ or $\boldsymbol{p}_{i(2)} \boldsymbol{p}_{i(2)+1}$ is a negative element.

Suppose that the lemma holds for $j-1$, i.e., $N_{j-1}+A_{j-1} \geq j-2$, with equality implying that $\boldsymbol{p}_{i(j)+1}$ or $\boldsymbol{p}_{i(j)} \boldsymbol{p}_{i(j)+1}$ is a negative element.

The inequality $N_{j}+A_{j} \geq j-1$ is then immediate, since either $N_{j-1}+A_{j-1}>j-2$, hence $N_{j}+A_{j} \geq N_{j-1}+A_{j-1} \geq j-1$, or $N_{j-1}+A_{j-1}=j-2$ and then there is at least one negative element between $\boldsymbol{p}_{i(j)}$ and $\boldsymbol{p}_{i(j+1)}$, i.e., $N_{j}>N_{j-1}$, hence $N_{j}+A_{j}>$ $N_{j-1}+A_{j-1}=j-2$.

Suppose now $N_{j}+A_{j}=j-1$. If $N_{j-1}+A_{j-1}>j-2$, then $N_{j}+A_{j}=N_{j-1}+A_{j-1}$, i.e., there is no negative element nor an arc of size at least $2 m-3$ between $\boldsymbol{p}_{i(j)}$ and $\boldsymbol{p}_{i(j+1)}$. By Lemma 6 it follows that $\boldsymbol{p}_{i(j+1)+1}$ or $\boldsymbol{p}_{i(j+1)} \boldsymbol{p}_{i(j+1)+1}$ is a negative element, which is what we required. In the remaining case $N_{j-1}+A_{j-1}=j-2$ and by the induction hypothesis $\boldsymbol{p}_{i(j)+1}$ or $\boldsymbol{p}_{i(j)} \boldsymbol{p}_{i(j)+1}$ is a negative element. Also, since now $N_{j}+A_{j}=$ $N_{j-1}+A_{j-1}+1$, we cannot also have an arc of size at least $2 m-3$ between $\boldsymbol{p}_{i(j)}$ and $\boldsymbol{p}_{i(j+1)}$. Again Lemma 6 gives the required conclusion.

Concluding the Proof of Lemma 5. We apply Lemma 7 to $j=d_{3}$. Thus $N+A \geq$ $d_{3}-1$, but equality cannot hold, since there is no negative element between $\boldsymbol{p}_{i\left(d_{3}+1\right)}=\boldsymbol{p}_{i(1)}$ and $\boldsymbol{p}_{i(2)}$ by our choice of $\boldsymbol{p}_{i(1)}$ and $\boldsymbol{p}_{i(2)}$.

We now fix a nonconcave arc of size exactly $2 m-3$ as follows: We let $\boldsymbol{p}_{1}, \ldots, \boldsymbol{p}_{2 m-3}$ be the degree 4 vertices with $\operatorname{deg}\left(\boldsymbol{p}_{0}\right)=3$ and let $\boldsymbol{p}_{0} \boldsymbol{p}_{1}, \boldsymbol{p}_{1} \boldsymbol{p}_{2}, \ldots, \boldsymbol{p}_{2 m-4} \boldsymbol{p}_{2 m-3}$ be all 


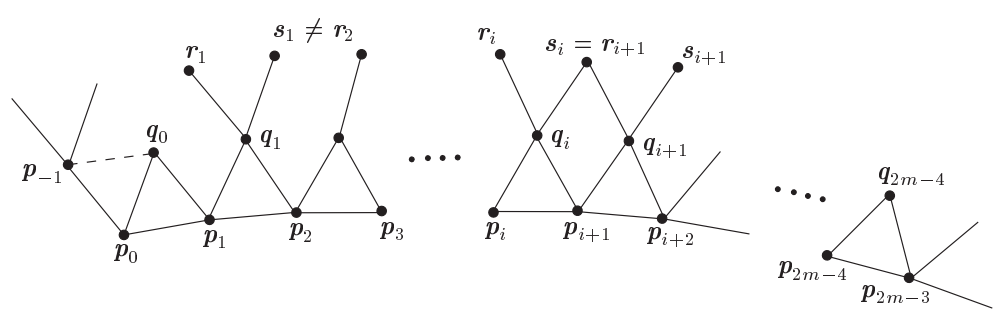

Fig. 4. Broken lattice.

triangle edges, and either $\operatorname{deg}\left(\boldsymbol{p}_{-1}\right)=3$, or $\operatorname{deg}\left(\boldsymbol{p}_{-1}\right)=4$ and $\boldsymbol{p}_{-1} \boldsymbol{p}_{0}$ a triangle edge. Recall that we denote the common neighbour of $\boldsymbol{p}_{i}$ and $\boldsymbol{p}_{i+1}$ by $\boldsymbol{q}_{i}(i=0, \ldots, 2 m-4)$. See Fig. 4.

Lemma 8. Each $\boldsymbol{q}_{i}(i=1, \ldots, 2 m-5)$ has exactly two neighbours other than $\boldsymbol{p}_{i}, \boldsymbol{p}_{i+1}$ or (possibly) $\boldsymbol{q}_{i-1}, \boldsymbol{q}_{i+1}$, which we denote by $\boldsymbol{r}_{i}$ and $\boldsymbol{s}_{i}$ in such a way that the points $\boldsymbol{s}_{i}, \boldsymbol{r}_{i}, \boldsymbol{q}_{i-1}, \boldsymbol{p}_{i}, \boldsymbol{p}_{i+1}, \boldsymbol{q}_{i+1}$ are in this positive order around $\boldsymbol{q}_{i}$.

Proof. Since the arc is nonconcave, we have $\tau_{i}+\tau_{i+1} \leq \tau_{1}+\cdots+\tau_{2 m-3}<\pi / 3$ (recall that $\tau_{j} \geq 0$ whenever $\operatorname{deg}\left(\boldsymbol{p}_{j}\right) \geq 4$ ). Suppose $\boldsymbol{q}_{i}$ has three neighbours $\boldsymbol{r}_{1}, \boldsymbol{r}_{2}, \boldsymbol{r}_{3}$ other than $\boldsymbol{q}_{i-1}, \boldsymbol{q}_{i+1}, \boldsymbol{p}_{i}$ or $\boldsymbol{p}_{i+1}$. See Fig. 5. Since by Proposition 4, property 2,

$$
\mu\left(\varangle \boldsymbol{r}_{1} \boldsymbol{q}_{i} \boldsymbol{r}_{2}\right), \mu\left(\varangle \boldsymbol{r}_{2} \boldsymbol{q}_{i} \boldsymbol{r}_{3}\right), \mu\left(\varangle \boldsymbol{p}_{i} \boldsymbol{q}_{i} \boldsymbol{p}_{i+1}\right) \geq \frac{\pi}{3},
$$

we obtain that $\mu\left(\varangle \boldsymbol{r}_{3} \boldsymbol{q}_{i} \boldsymbol{p}_{i}\right)+\mu\left(\varangle \boldsymbol{p}_{i+1} \boldsymbol{q}_{i} \boldsymbol{r}_{1}\right) \leq \pi$. By Proposition 4, property 3,

$$
\mu\left(\varangle \boldsymbol{q}_{i} \boldsymbol{p}_{i} \boldsymbol{q}_{i-1}\right)+\mu\left(\varangle \boldsymbol{r}_{3} \boldsymbol{q}_{i} \boldsymbol{p}_{i}\right) \geq \pi \quad \text { and } \quad \mu\left(\varangle \boldsymbol{q}_{i+1} \boldsymbol{p}_{i+1} \boldsymbol{q}_{i}\right)+\mu\left(\varangle \boldsymbol{p}_{i+1} \boldsymbol{q}_{i} \boldsymbol{r}_{1}\right) \geq \pi \text {. }
$$

It follows that $\mu\left(\varangle \boldsymbol{q}_{i} \boldsymbol{p}_{i} \boldsymbol{q}_{i-1}\right)+\mu\left(\varangle \boldsymbol{q}_{i+1} \boldsymbol{p}_{i+1} \boldsymbol{q}_{i}\right) \geq \pi$, hence

$$
\begin{aligned}
\tau_{i}+\tau_{i+1} & =\theta_{i}+\theta_{i+1}-2 \pi \\
& =\mu\left(\varangle \boldsymbol{q}_{i} \boldsymbol{p}_{i} \boldsymbol{q}_{i-1}\right)+\frac{2 \pi}{3}+\mu\left(\varangle \boldsymbol{q}_{i+1} \boldsymbol{p}_{i+1} \boldsymbol{q}_{i}\right)+\frac{2 \pi}{3}-2 \pi \\
& \geq \frac{\pi}{3},
\end{aligned}
$$

a contradiction.

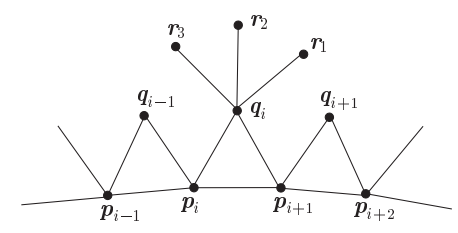

Fig. 5. Proof of Lemma 8. 
Suppose now $\boldsymbol{q}_{i}$ has at most one neighbour other than $\boldsymbol{q}_{i-1}, \boldsymbol{q}_{i+1}, \boldsymbol{p}_{i}$ or $\boldsymbol{p}_{i+1}$. Then the $\lfloor i / 2\rfloor+3=: k$ points $\boldsymbol{q}_{i}, \boldsymbol{p}_{i+2}, \boldsymbol{p}_{i-2 h-1}(h=0,1, \ldots,\lfloor i / 2\rfloor)$ have at most $3 k-1$ neighbours. Since $k \leq\lfloor(2 m-5) / 2\rfloor+3=m$, Lemma 1 will give a contradiction if we can show that these points are distinct and independent. By exactly the same reasoning as in the proof of Lemma 6 we have that $\boldsymbol{p}_{i+2}, \boldsymbol{p}_{i-2 h-1}(0 \leq h \leq\lfloor i / 2\rfloor)$ are distinct and independent. If $\boldsymbol{q}_{i}=\boldsymbol{p}_{i-2 h-1}$ for some $h$, then in the polygon $\boldsymbol{p}_{i} \boldsymbol{p}_{i-1} \boldsymbol{p}_{i-2} \cdots \boldsymbol{p}_{i-2 h-1}$ only the vertices $\boldsymbol{p}_{i}$ and $\boldsymbol{q}_{i}$ can possibly have degree 3 in the subgraph induced by the polygon and its interior, contradicting Lemma 4 . Clearly $\boldsymbol{q}_{i}$ and $\boldsymbol{p}_{i+2}$ are distinct neighbours of $\boldsymbol{p}_{i+1}$, and, similarly, $\boldsymbol{q}_{i} \neq \boldsymbol{p}_{i-1}$. Since $\boldsymbol{p}_{i+1}$ and $\boldsymbol{p}_{i+2}$ have only one common neighbour, $\boldsymbol{q}_{i}$ is not adjacent to $\boldsymbol{p}_{i+2}$. Similarly, $\boldsymbol{q}_{i}$ is not adjacent to $\boldsymbol{p}_{i-1}$. If $\boldsymbol{q}_{i}$ is adjacent to some $\boldsymbol{p}_{i-2 h-1}$, then in the polygon $\boldsymbol{q}_{i} \boldsymbol{p}_{i} \boldsymbol{p}_{i-1} \boldsymbol{p}_{i-2} \cdots \boldsymbol{p}_{i-2 h-1}$ only the vertices $\boldsymbol{q}_{i}, \boldsymbol{p}_{i}$ and $\boldsymbol{p}_{i-2 h-1}$ can possibly have degrees of at most 3 in the subgraph induced by the polygon and its interior with $\boldsymbol{p}_{i}$ and $\boldsymbol{p}_{i-2 h-1}$ having degree exactly 3 , again contradicting Lemma 4 . Thus the set is independent, giving the required contradiction.

It follows that $\boldsymbol{q}_{i}$ must have exactly two neighbours.

Lemma 9. If $\boldsymbol{s}_{i}=\boldsymbol{r}_{i+1}$ for each $i=a, a+1, a+2$ for some $1 \leq a \leq 2 m-8$, then $a \geq 2 m-10$.

Proof. See Fig. 6. By using an angle estimation as in the proof of Lemma 8 one can show that if $\boldsymbol{s}_{a+1}$ has more than two neighbours other than $\boldsymbol{s}_{a}, \boldsymbol{s}_{a+2}, \boldsymbol{q}_{a+1}$ or $\boldsymbol{q}_{a+2}$, then $\tau_{a+1}+\tau_{a+2}+\tau_{a+3} \geq \pi / 3$, a contradiction. Thus there are at most two. Then the $\lfloor a / 2\rfloor+6=: k$ points $\boldsymbol{p}_{a+5}, \boldsymbol{q}_{a+3}, \boldsymbol{p}_{a+2}, \boldsymbol{s}_{a+1}, \boldsymbol{q}_{a}, \boldsymbol{p}_{a-2 h-1}(h=0,1, \ldots,\lfloor a / 2\rfloor)$ have at most $3 k-1$ neighbours. By Lemma 1 we must have $k>m$, which is equivalent to $a \geq 2 m-10$, if we can show that these $k$ points are distinct and independent.

As in the proof of Lemma 8 the points $\boldsymbol{p}_{a+5}, \boldsymbol{p}_{a+2}, \boldsymbol{p}_{a-2 h-1}$ are all distinct and independent, and are not equal or adjacent to $\boldsymbol{q}_{a}$ or $\boldsymbol{q}_{a+3}$.

If $\boldsymbol{q}_{a}=\boldsymbol{q}_{a+3}$, then in the subgraph induced by the polygon $\boldsymbol{q}_{a} \boldsymbol{p}_{a+1} \boldsymbol{p}_{a+2} \boldsymbol{p}_{a+3}$ and its interior there are only two points of degree 3 and one of degree 2, contradicting Lemma 4 . If $\boldsymbol{q}_{a}$ is adjacent to $\boldsymbol{q}_{a+3}$, then we consider the polygon $\boldsymbol{q}_{a} \boldsymbol{p}_{a+1} \boldsymbol{p}_{a+2} \boldsymbol{p}_{a+3} \boldsymbol{q}_{a+3}$ and take care of the possible neighbours of $\boldsymbol{q}_{a}$ in turn: If $\boldsymbol{q}_{a+3}=\boldsymbol{q}_{a-1}$, then only $\boldsymbol{p}_{a+1}$ and $\boldsymbol{p}_{a+3}$ have degree 3 and only $\boldsymbol{q}_{a+3}$ has degree 2 . If $\boldsymbol{q}_{a+3}=\boldsymbol{r}_{a}$, then only $\boldsymbol{p}_{a+1}, \boldsymbol{p}_{a+3}$ and $\boldsymbol{q}_{a}$ have degree 3 and only $\boldsymbol{q}_{a+3}$ has degree 2 . The case $\boldsymbol{q}_{a+3}=\boldsymbol{q}_{a+1}$ is ruled out since $\boldsymbol{p}_{a+2}$ and $\boldsymbol{p}_{a+3}$ have only one common neighbour. The cases $\boldsymbol{q}_{a+3}=\boldsymbol{p}_{a}$ and $\boldsymbol{p}_{a+1}$ are ruled out as before.

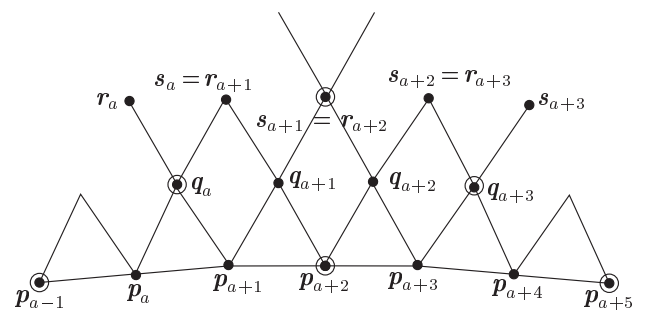

Fig. 6. Proof of Lemma 9. 
By definition, $\boldsymbol{s}_{a+1} \neq \boldsymbol{q}_{a}$ and $\boldsymbol{r}_{a+2} \neq \boldsymbol{q}_{a+3}$. By Proposition 5, $\boldsymbol{s}_{a+1}$ is not adjacent to $\boldsymbol{q}_{a}$ nor $\boldsymbol{q}_{a+3}$, since $\boldsymbol{s}_{a}$ and $\boldsymbol{p}_{a+1}$ are already common neighbours of $\boldsymbol{q}_{a}$ and $\boldsymbol{q}_{a+1}$, and $\boldsymbol{s}_{a+2}$ and $\boldsymbol{p}_{a+3}$ are already common neighbours of $\boldsymbol{q}_{a+2}$ and $\boldsymbol{q}_{a+3}$.

If $\boldsymbol{s}_{a+1}$ is equal or adjacent to $\boldsymbol{p}_{a+5}$, then Lemma 4 applied to the polygon

$$
\boldsymbol{s}_{a+1} \boldsymbol{q}_{a+1} \boldsymbol{p}_{a+2} \boldsymbol{p}_{a+3} \boldsymbol{p}_{a+4} \boldsymbol{p}_{a+5}
$$

as before gives a contradiction. By definition of $\boldsymbol{s}_{a+1}$ we have $\boldsymbol{s}_{a+1} \neq \boldsymbol{p}_{a+2}$. If $\boldsymbol{s}_{a+1}$ and $\boldsymbol{p}_{a+2}$ are adjacent, then $\boldsymbol{s}_{a+1}=\boldsymbol{p}_{a+1}$ or $\boldsymbol{s}_{a+1}=\boldsymbol{p}_{a+3}$, contradicting the definitions of $\boldsymbol{s}_{a+1}$ and $\boldsymbol{r}_{a+2}$. Finally, if $\boldsymbol{s}_{a+1}$ is equal or adjacent to some $\boldsymbol{p}_{a-2 h-1}$, then Lemma 4 applied to the polygon $\boldsymbol{s}_{a+1} \boldsymbol{q}_{a+1} \boldsymbol{p}_{a+1} \boldsymbol{p}_{a} \boldsymbol{p}_{a-1} \cdots \boldsymbol{p}_{a-2 h-1}$ gives a contradiction as before.

We can summarize the results of this section in the following technical theorem.

Theorem 4. Let $m \geq 5$ and let $G$ be a minimum distance graph $G$ on $n$ points in a nonrectilinear normed plane with an arbitrary Brass measure $\mu$. If $G$ is a smallest counterexample to $\alpha(G) \geq(m /(4 m-1)) n$, then $G$ is planar two-connected and there is a consecutive sequence $\boldsymbol{p}_{0}, \boldsymbol{p}_{1}, \ldots, \boldsymbol{p}_{2 m-3}$ of vertices on the boundary polygon such that

1. the total turn $\tau_{1}+\cdots+\tau_{2 m-3}<\pi / 3$,

2. $\operatorname{deg}\left(\boldsymbol{p}_{0}\right)=3$ and $\operatorname{deg}\left(\boldsymbol{p}_{i}\right)=4(i=1, \ldots, 2 m-3)$,

3. $\boldsymbol{p}_{i}$ and $\boldsymbol{p}_{i+1}$ have a unique common neighbour $\boldsymbol{q}_{i}(i=0, \ldots, 2 m-4)$,

4. each $\boldsymbol{q}_{i}(i=2, \ldots, 2 m-5)$ has exactly two neighbours not in $\left\{\boldsymbol{q}_{i-1}, \boldsymbol{q}_{i+1}, \boldsymbol{p}_{i}, \boldsymbol{p}_{i+1}\right\}$, denoted by $\boldsymbol{r}_{i}$ and $\boldsymbol{s}_{i}$ in such a way that the points $\boldsymbol{s}_{i}, \boldsymbol{r}_{i}, \boldsymbol{q}_{i-1}, \boldsymbol{p}_{i}, \boldsymbol{p}_{i+1}, \boldsymbol{q}_{i+1}$ are in this positive order around $\boldsymbol{q}_{i}$,

5. If $\boldsymbol{s}_{i}=\boldsymbol{r}_{i+1}$ for each $i=a, a+1, a+2$ for some $a \geq 1$, then $a \geq 2 m-10$.

We saw that the proof used a minimum of geometric information specific to a particular normed plane. We now use more geometric properties to show that the above obstruction is impossible for sufficiently large $m$, first in the Euclidean plane (Section 4) and then in any plane where the unit ball is not a paralleloid (Section 5).

\section{The Euclidean Plane: Proof of Theorem 1}

We still consider the nonconcave arc $\boldsymbol{p}_{1}, \ldots, \boldsymbol{p}_{2 m-3}$ as in the previous section, together with the associated points $\boldsymbol{q}_{i}, \boldsymbol{r}_{i}, \boldsymbol{s}_{i}$. For the angular measure we now take the usual Euclidean angle.

Lemma 10. In the Euclidean plane we have $\boldsymbol{s}_{i}=\boldsymbol{r}_{i+1}$ for all $i=1, \ldots, 2 m-6$, except at most one.

Proof. It is sufficient to show for each $i=1, \ldots, 2 m-6$ that if $\boldsymbol{s}_{i} \neq \boldsymbol{r}_{i+1}$, then

$$
\tau_{i}+2 \tau_{i+1}+\tau_{i+2} \geq \frac{\pi}{3}
$$




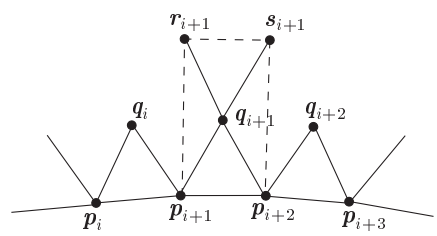

Fig. 7. Proof of Lemma 10.

and

$$
\tau_{i}+\tau_{i+1} \geq \mu\left(\varangle \boldsymbol{q}_{i+1} \boldsymbol{p}_{i+1} \boldsymbol{r}_{i+1}\right) .
$$

Sufficiency is seen as follows. Suppose that $\boldsymbol{s}_{i} \neq \boldsymbol{r}_{i+1}$ and $\boldsymbol{s}_{j} \neq \boldsymbol{r}_{j+1}$ for some $1 \leq i<$ $j \leq 2 m-6$. Recall that $\tau_{1}, \tau_{2}, \ldots, \tau_{2 m-3} \geq 0, \operatorname{since} \operatorname{deg}\left(\boldsymbol{p}_{1}\right)=\cdots=\operatorname{deg}\left(\boldsymbol{p}_{2 m-3}\right)=4$. Then in the case $i+2 \leq j$ we have by nonconcavity

$$
\begin{aligned}
\frac{\pi}{3} & > \begin{cases}\tau_{i}+\tau_{i+1}+\tau_{i+2}+\tau_{j}+\tau_{j+1}+\tau_{j+2} & \text { if } i+2<j \\
\tau_{i}+\tau_{i+1}+\tau_{i+2}+\tau_{j+1}+\tau_{j+2} & \text { if } i+2=j\end{cases} \\
& \geq \frac{1}{2}\left(\tau_{i}+2 \tau_{i+1}+\tau_{i+2}\right)+\frac{1}{2}\left(\tau_{j}+2 \tau_{j+1}+\tau_{j+2}\right)
\end{aligned}
$$

a contradiction.

In the case $i+1=j$ (Fig. 7) we have

$$
\begin{aligned}
\frac{\pi}{3} & >\tau_{i}+\tau_{i+1}+\tau_{i+2}+\tau_{i+3} \\
& \geq \mu\left(\varangle \boldsymbol{q}_{i+1} \boldsymbol{p}_{i+1} \boldsymbol{r}_{i+1}\right)+\mu\left(\varangle \boldsymbol{s}_{i+1} \boldsymbol{p}_{i+2} \boldsymbol{q}_{i+1}\right) \quad \text { (using (6) and its mirror). }
\end{aligned}
$$

Since

- $\triangle \boldsymbol{q}_{i+1} \boldsymbol{r}_{i+1} \boldsymbol{p}_{i+1}$ and $\triangle \boldsymbol{q}_{i+1} \boldsymbol{p}_{i+2} \boldsymbol{s}_{i+1}$ are isosceles,

- $\triangle \boldsymbol{p}_{i+1} \boldsymbol{p}_{i+2} \boldsymbol{q}_{i+1}$ is equilateral,

- the angle sum of $\triangle \boldsymbol{q}_{i+1} \boldsymbol{s}_{i+1} \boldsymbol{r}_{i+1}$ is $\pi$, and

- the angle sum of the quadrilateral $\boldsymbol{p}_{i+1} \boldsymbol{p}_{i+2} \boldsymbol{s}_{i+1} \boldsymbol{r}_{i+1}$ is $2 \pi$,

we obtain that

$$
\mu\left(\varangle \boldsymbol{q}_{i+1} \boldsymbol{p}_{i+1} \boldsymbol{r}_{i+1}\right)+\mu\left(\varangle \boldsymbol{s}_{i+1} \boldsymbol{p}_{i+2} \boldsymbol{q}_{i+1}\right)=\frac{1}{2}\left(\frac{\pi}{3}+\mu\left(\varangle \boldsymbol{s}_{i+1} \boldsymbol{q}_{i+1} \boldsymbol{r}_{i+1}\right)\right) .
$$

Since $\boldsymbol{r}_{i+1} \boldsymbol{s}_{i+1} \geq \boldsymbol{r}_{i+1} \boldsymbol{q}_{i+1}=\boldsymbol{q}_{i+1} \boldsymbol{s}_{i+1}$ we have $\mu\left(\varangle \boldsymbol{s}_{i+1} \boldsymbol{q}_{i+1} \boldsymbol{r}_{i+1}\right) \geq \pi / 3$, and it follows from (7) and (8) that $\tau_{i}+\tau_{i+1}+\tau_{i+2}+\tau_{i+3} \geq \pi / 3$, a contradiction.

Proof of (5). Let $\alpha=\mu\left(\varangle \boldsymbol{q}_{i+1} \boldsymbol{p}_{i+1} \boldsymbol{q}_{i}\right), \alpha^{\prime}=\mu\left(\varangle \boldsymbol{q}_{i} \boldsymbol{p}_{i} \boldsymbol{q}_{i-1}\right), \alpha^{\prime \prime}=\mu\left(\varangle \boldsymbol{q}_{i+2} \boldsymbol{p}_{i+2} \boldsymbol{q}_{i+1}\right)$, $\beta=\mu\left(\varangle \boldsymbol{p}_{i+1} \boldsymbol{q}_{i} \boldsymbol{s}_{i}\right), \beta^{\prime}=\mu\left(\varangle \boldsymbol{r}_{i} \boldsymbol{q}_{i} \boldsymbol{p}_{i}\right), \gamma=\mu\left(\varangle \boldsymbol{q}_{i} \boldsymbol{s}_{i} \boldsymbol{r}_{i+1}\right), \delta=\mu\left(\varangle \boldsymbol{s}_{i} \boldsymbol{r}_{i+1} \boldsymbol{q}_{i+1}\right), \varepsilon=$ $\mu\left(\varangle \boldsymbol{r}_{i+1} \boldsymbol{q}_{i+1} \boldsymbol{p}_{i+1}\right), \varepsilon^{\prime}=\mu\left(\varangle \boldsymbol{p}_{i+2} \boldsymbol{q}_{i+1} \boldsymbol{s}_{i+1}\right), d_{1}=\boldsymbol{s}_{i} \boldsymbol{r}_{i+1}$ and $d_{2}=\boldsymbol{q}_{i} \boldsymbol{q}_{i+1}$ (Fig. 8(a)). 


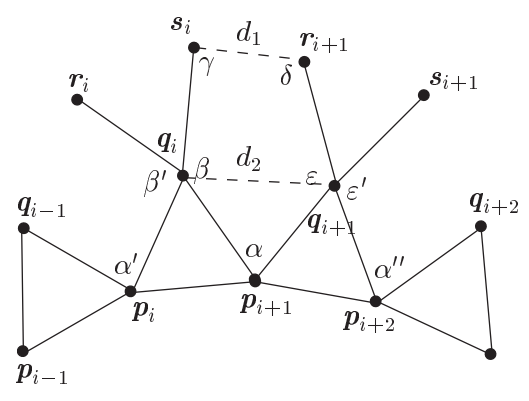

(a)

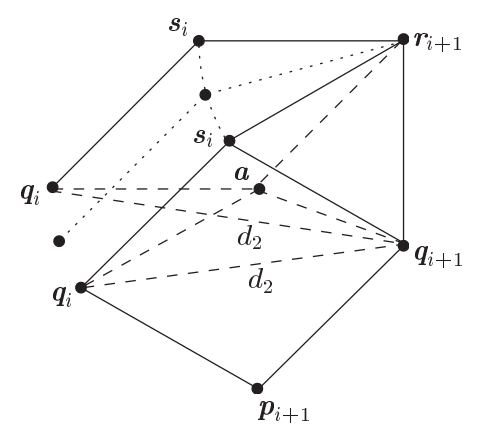

(b)

Fig. 8. Proof of (5).

The left-hand side of (5) is

$$
\begin{aligned}
\tau_{i}+2 \tau_{i+1}+\tau_{i+2} & =\theta_{i}-\pi+2\left(\theta_{i+1}-\pi\right)+\theta_{i+2}-\pi \\
& =\alpha^{\prime}+2 \alpha+\alpha^{\prime \prime}-\frac{4 \pi}{3} \\
& \geq\left(\pi-\beta^{\prime}\right)+2 \alpha+\left(\pi-\varepsilon^{\prime}\right)-\frac{4 \pi}{3} \quad \text { (Proposition 4, property 3) } \\
& =2 \alpha-\beta^{\prime}-\varepsilon^{\prime}+\frac{2 \pi}{3} \\
& \geq 2 \alpha-\left(\frac{4 \pi}{3}-\beta\right)-\left(\frac{4 \pi}{3}-\varepsilon\right)+\frac{2 \pi}{3} \\
& \quad\left(\text { since } \mu\left(\varangle s_{i} \boldsymbol{q}_{i} \boldsymbol{r}_{i}\right), \mu\left(\varangle \boldsymbol{s}_{i+1} \boldsymbol{q}_{i+1} \boldsymbol{r}_{i+1}\right) \geq \frac{\pi}{3}\right) \\
& =2 \alpha+\beta+\varepsilon-2 \pi \quad(3 \pi-\alpha-\gamma-\delta)-2 \pi \\
& =2 \alpha+\left(\text { angle sum of pentagon } \boldsymbol{p}_{i+1} \boldsymbol{q}_{i+1} \boldsymbol{r}_{i+1} \boldsymbol{s}_{i} \boldsymbol{q}_{i}\right) \\
& =\alpha-\gamma-\delta+\pi .
\end{aligned}
$$

To prove (5) it is therefore sufficient to prove that $\gamma+\delta \leq \alpha+2 \pi / 3$.

Since $\gamma+\delta$ is a decreasing function of $d_{1}$ under the constraints $\boldsymbol{q}_{i} \boldsymbol{s}_{i}=\boldsymbol{r}_{i+1} \boldsymbol{q}_{i+1}=$ $1, \boldsymbol{q}_{i} \boldsymbol{q}_{i+1}=d_{2}$, we may assume without loss of generality that $d_{1}=1$. Also, $\alpha$ is an increasing function of $d_{2}$ under the constraints $\boldsymbol{p}_{i+1} \boldsymbol{q}_{i}=\boldsymbol{p}_{i+1} \boldsymbol{q}_{i+1}=1$. Thus it is sufficient to show that for fixed $\gamma+\delta, d_{2}$ is minimized under the constraints $\boldsymbol{q}_{i} \boldsymbol{s}_{i}=$ $\boldsymbol{s}_{i} \boldsymbol{r}_{i+1}=\boldsymbol{r}_{i+1} \boldsymbol{q}_{i+1}=1$ when $\alpha+2 \pi / 3=\gamma+\delta$. We fix the points $\boldsymbol{r}_{i+1}$ and $\boldsymbol{q}_{i+1}$, and translate the segment $\boldsymbol{s}_{i} \boldsymbol{q}_{i}$ such that $\boldsymbol{s}_{i} \boldsymbol{r}_{i+1}$ remains a unit segment. See Fig. 8(b). Then $\gamma+\delta$ stays constant. Also note that $\boldsymbol{a}:=\boldsymbol{r}_{i+1}+\boldsymbol{q}_{i}-\boldsymbol{s}_{i}$ is a constant, hence the lengths $\boldsymbol{q}_{i} \boldsymbol{a}$ and $\boldsymbol{a} \boldsymbol{q}_{i+1}$ are constants, while $d_{2}$ will be minimized if $\mu\left(\varangle \boldsymbol{q}_{i+1} \boldsymbol{a q _ { i }}\right)$ is as small as possible. This happens when $\delta$ is as small as possible, i.e., when $\delta=\pi / 3$. Then $\Delta \boldsymbol{r}_{i+1} \boldsymbol{s}_{i} \boldsymbol{q}_{i+1}$ is equilateral and $\boldsymbol{s}_{i} \boldsymbol{q}_{i+1} \boldsymbol{p}_{i+1} \boldsymbol{q}_{i}$ is a rhombus, hence $\gamma=\mu\left(\varangle \boldsymbol{q}_{i+1} \boldsymbol{s}_{i} \boldsymbol{q}_{i}\right)+\pi / 3=\alpha+\pi / 3$ and $\gamma+\delta=\alpha+2 \pi / 3$, as required. 


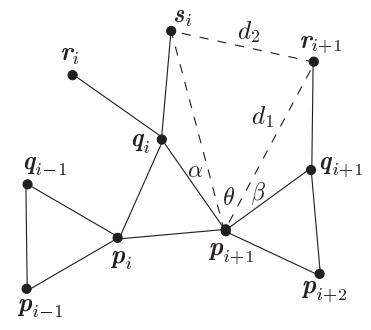

Fig. 9. Proof of (6).

Proof of (6). Let $\alpha=\mu\left(\varangle \boldsymbol{s}_{i} \boldsymbol{p}_{i+1} \boldsymbol{q}_{i}\right), \theta=\mu\left(\varangle \boldsymbol{r}_{i+1} \boldsymbol{p}_{i+1} \boldsymbol{s}_{i}\right), \beta=\mu\left(\varangle \boldsymbol{q}_{i+1} \boldsymbol{p}_{i+1} \boldsymbol{r}_{i+1}\right)$, $d_{1}=\boldsymbol{p}_{i+1} \boldsymbol{r}_{i+1}$ and $d_{2}=\boldsymbol{s}_{i} \boldsymbol{r}_{i+1}$ (Fig. 9). We have

$$
\begin{aligned}
\tau_{i+1} & =\theta_{i+1}-\pi=\mu\left(\varangle \boldsymbol{q}_{i+1} \boldsymbol{p}_{i+1} \boldsymbol{q}_{i}\right)-\frac{\pi}{3} \\
& =\alpha+\theta+\beta-\frac{\pi}{3}
\end{aligned}
$$

and, similarly,

$$
\begin{aligned}
\tau_{i} & =\mu\left(\varangle \boldsymbol{q}_{i} \boldsymbol{p}_{i} \boldsymbol{q}_{i-1}\right)-\frac{\pi}{3} \\
& \geq \pi-\mu\left(\varangle \boldsymbol{r}_{i} \boldsymbol{q}_{i} \boldsymbol{p}_{i}\right)-\frac{\pi}{3} \quad \text { (Proposition 4, property 3) } \\
& =\frac{2 \pi}{3}-\left(2 \pi-\mu\left(\varangle \boldsymbol{p}_{i} \boldsymbol{q}_{i} \boldsymbol{p}_{i+1}\right)-\mu\left(\varangle \boldsymbol{p}_{i+1} \boldsymbol{q}_{i} \boldsymbol{s}_{i}\right)-\mu\left(\varangle \boldsymbol{s}_{i} \boldsymbol{q}_{i} \boldsymbol{r}_{i}\right)\right) \\
& =\frac{2 \pi}{3}-\left(2 \pi-\frac{\pi}{3}-\pi+2 \alpha-\mu\left(\varangle s_{i} \boldsymbol{q}_{i} \boldsymbol{r}_{i}\right)\right)
\end{aligned}
$$

since $\triangle \boldsymbol{p}_{i} \boldsymbol{p}_{i+1} \boldsymbol{q}_{i}$ is equilateral and $\triangle \boldsymbol{q}_{i} \boldsymbol{p}_{i+1} \boldsymbol{s}_{i}$ is isosceles, hence

$$
\tau_{i} \geq \frac{\pi}{3}-2 \alpha
$$

since $\boldsymbol{r}_{i} \boldsymbol{s}_{i} \geq \boldsymbol{r}_{i} \boldsymbol{q}_{i}=\boldsymbol{s}_{i} \boldsymbol{q}_{i}$. This estimate is better than $\tau_{i} \geq 0$ iff $\alpha \leq \pi / 6$. We now consider the two cases $\alpha \leq \pi / 6$ and $\alpha \geq \pi / 6$ separately. Note that in general $\alpha \leq \pi / 3$, since $\mu\left(\varangle \boldsymbol{p}_{i+1} \boldsymbol{q}_{i} \boldsymbol{s}_{i}\right) \geq \pi / 3$.

In the case $\alpha \leq \pi / 6$ we have, adding (9) and (10), that $\tau_{i}+\tau_{i+1} \geq \beta+\theta-\alpha$, hence to prove (6) it is sufficient to show that $\theta \geq \alpha$, which is equivalent to $\cos \theta \leq \cos \alpha$, since $\alpha \leq \pi / 6<\pi$. It follows from $\boldsymbol{s}_{i} \boldsymbol{p}_{i+1}=2 \cos \alpha$, the cosine rule in $\triangle \boldsymbol{s}_{i} \boldsymbol{p}_{i+1} \boldsymbol{r}_{i+1}$ and the inequalities $d_{2} \geq 1$ and $\alpha<\pi / 2$ that

$$
\cos \theta \leq \frac{d_{1}^{2}-1+4 \cos ^{2} \alpha}{4 d_{1} \cos \alpha} .
$$

It is then sufficient to show that

$$
\frac{d_{1}^{2}-1+4 \cos ^{2} \alpha}{4 d_{1} \cos \alpha} \leq \cos \alpha,
$$


which is equivalent to $d_{1}^{2}-1 \leq 4\left(d_{1}-1\right) \cos ^{2} \alpha$. Since this inequality trivially holds for $d_{1}=1$ and also $d_{1} \geq 1$ in general, it is sufficient to prove that $d_{1}+1 \leq 4 \cos ^{2} \alpha$. However, this holds since $d_{1} \leq \boldsymbol{p}_{i+1} \boldsymbol{q}_{i+1}+\boldsymbol{q}_{i+1} \boldsymbol{r}_{i+1} \leq 2$ and $\alpha \leq \pi / 6$.

In the case $\alpha \geq \pi / 6$ we estimate as follows:

$$
\tau_{i}+\tau_{i+1} \geq \tau_{i+1}=\alpha+\theta+\beta-\frac{\pi}{3} \quad(\text { by }(9)),
$$

hence to prove (6) it is sufficient to show that $\alpha+\theta \geq \pi / 3$, which (since $\alpha \leq \pi / 3<\pi$ ) is equivalent to $\cos \theta \leq \frac{1}{2} \cos \alpha+(\sqrt{3} / 2) \sin \alpha$. Since inequality (11) still holds, it is sufficient to show that

$$
\frac{d_{1}^{2}-1+4 \cos ^{2} \alpha}{4 d_{1} \cos \alpha} \leq \frac{1}{2} \cos \alpha+\frac{\sqrt{3}}{2} \sin \alpha
$$

which is equivalent to

$$
d_{1}^{2}-1+\left(2-d_{1}\right)(1+\cos 2 \alpha) \leq \sqrt{3} d_{1} \sin 2 \alpha .
$$

Since $\alpha \geq \pi / 6$ we have $\cos 2 \alpha \leq \frac{1}{2}$ and $\sqrt{3} / 2 \leq \sin 2 \alpha$. Since also $2-d_{1} \geq 0$, it is sufficient to prove that

$$
d_{1}^{2}-1+\left(2-d_{1}\right)\left(1+\frac{1}{2}\right) \leq \sqrt{3} d_{1} \frac{\sqrt{3}}{2},
$$

which follows from $1 \leq d_{1} \leq 2$.

Conclusion of the Proof of Theorem 1. We apply Theorem 4 with $m=8$. By Lemma 10 we have that $\boldsymbol{s}_{i}=\boldsymbol{r}_{i+1}$ for each $i=a, a+1, a+2$ for some $a \leq 4$. However, by Theorem 4 we must have $a \geq 2 m-10=6$ in a smallest counterexample. Thus there are no counterexamples to $F(n) \geq(m /(4 m-1)) n=\frac{8}{31} n$.

\section{The Nonparalleloid Case: Proof of Theorem 2}

As in the previous section we consider the nonconcave $\operatorname{arc} \boldsymbol{p}_{1}, \ldots, \boldsymbol{p}_{2 m-3}$ together with the points $\boldsymbol{q}_{i}, \boldsymbol{r}_{i}, \boldsymbol{s}_{i}$. We assume that the unit ball $C$ of $M(C)$ is not a paralleloid, and we fix a Brass measure $\mu$. To find an appropriately large value of $m$ we first analyse the nonconcave arc locally.

Lemma 11. For each $\varepsilon>0$ there exists $\delta>0$ such that in the minimum distance graph in Fig. 10, if $\mu(\varangle \boldsymbol{r q p}), \mu\left(\varangle \boldsymbol{p}_{+} \boldsymbol{q} \boldsymbol{s}\right)>2 \pi / 3-\delta$, then

$$
\left\|\frac{\boldsymbol{r}-\boldsymbol{p}}{\|\boldsymbol{r}-\boldsymbol{p}\|}-\frac{\boldsymbol{s}-\boldsymbol{p}_{+}}{\left\|\boldsymbol{s}-\boldsymbol{p}_{+}\right\|}\right\|<\varepsilon
$$

Proof. If the lemma is false, for some $\varepsilon>0$ we can find sequences $\boldsymbol{p}^{n}, \boldsymbol{p}_{+}^{n}, \boldsymbol{q}^{n}, \boldsymbol{r}^{n}, \boldsymbol{s}^{n}$ of points such that $\mu\left(\varangle \boldsymbol{r}^{n} \boldsymbol{q}^{n} \boldsymbol{p}^{n}\right), \mu\left(\varangle \boldsymbol{p}_{+}^{n} \boldsymbol{q}^{n} \boldsymbol{s}^{n}\right)>2 \pi / 3-1 / n$, and setting $\boldsymbol{x}^{n}=\left(\boldsymbol{r}^{n}-\right.$ 


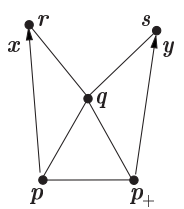

Fig. 10. Local analysis of nonconcave arc in Lemma 11.

$\left.\boldsymbol{p}^{n}\right) /\left\|\boldsymbol{r}^{n}-\boldsymbol{p}^{n}\right\|$ and $\boldsymbol{y}^{n}=\left(\boldsymbol{s}^{n}-\boldsymbol{p}^{n}\right) /\left\|\boldsymbol{s}^{n}-\boldsymbol{p}^{n}\right\|$, we also have $\left\|\boldsymbol{x}^{n}-\boldsymbol{y}^{n}\right\| \geq \varepsilon$. Since for each $n$ the set $\left\{\boldsymbol{p}^{n}, \boldsymbol{p}_{+}^{n}, \boldsymbol{q}^{n}, \boldsymbol{r}^{n}, \boldsymbol{s}^{n}\right\}$ has diameter at most 2, we may assume that all the sequences are contained in a bounded region of the plane. Thus we may take a convergent subsequence and in the limit obtain points $\boldsymbol{p}, \boldsymbol{p}_{+}, \boldsymbol{q}, \boldsymbol{r}, \boldsymbol{s}, \boldsymbol{x}, \boldsymbol{y}$ with $\mu(\varangle \boldsymbol{r q p}), \mu\left(\varangle \boldsymbol{p}_{+} \boldsymbol{q} \boldsymbol{s}\right) \geq$ $2 \pi / 3$, and $\|\boldsymbol{x}-\boldsymbol{y}\| \geq \varepsilon$. However, $\mu(\varangle \boldsymbol{s q r}) \geq \pi / 3=\mu\left(\varangle \boldsymbol{p q p} \boldsymbol{p}_{+}\right)$, hence $\mu(\varangle \boldsymbol{r q p})=$ $\mu\left(\varangle \boldsymbol{p}_{+} \boldsymbol{q} \boldsymbol{s}\right)=2 \pi / 3$.

Since $\mu$ is proper, we then have that $\boldsymbol{r}, \boldsymbol{q}, \boldsymbol{p}_{+}$are collinear, hence $\boldsymbol{r}-\boldsymbol{q}=\boldsymbol{q}-\boldsymbol{p}_{+}$and, similarly, $\boldsymbol{s}, \boldsymbol{q}, \boldsymbol{p}$ are collinear, hence $\boldsymbol{s}-\boldsymbol{q}=\boldsymbol{q}-\boldsymbol{p}$. Thus $\boldsymbol{x}=\boldsymbol{r}-\boldsymbol{p}=\boldsymbol{r}-\boldsymbol{q}+\boldsymbol{q}-\boldsymbol{p}=$ $\boldsymbol{q}-\boldsymbol{p}_{+}+\boldsymbol{s}-\boldsymbol{q}=\boldsymbol{s}-\boldsymbol{p}_{+}=\boldsymbol{y}$, a contradiction.

We omit the proofs of the following two lemmas, which are used repeatedly in the proof of Lemma 14.

Lemma 12. In a normed plane in which the unit ball is not a paralleloid, let $\boldsymbol{u}$ and $\boldsymbol{v}$ be unit vectors with $\boldsymbol{u v}=1$.

1. If $\boldsymbol{x}$ is a unit vector strictly between $\boldsymbol{u}$ and $\boldsymbol{v}$, then $\boldsymbol{u x}<1$.

2. If $\boldsymbol{x}$ is a unit vector strictly between $\boldsymbol{v}$ and $\boldsymbol{v}-\boldsymbol{u}$, then $\boldsymbol{u} \boldsymbol{x}>1$.

Lemma 13. In a normed plane let $\boldsymbol{u}$ and $\boldsymbol{v}$ be unit vectors with $\boldsymbol{u} \boldsymbol{v}=1$. Let $\boldsymbol{x}_{1}=$ $-\boldsymbol{x}_{4}=\boldsymbol{u}, \boldsymbol{x}_{2}=-\boldsymbol{x}_{5}=\boldsymbol{v}, \boldsymbol{x}_{3}=-\boldsymbol{x}_{0}=\boldsymbol{v}-\boldsymbol{u}$, and let $\boldsymbol{y}_{i}=\boldsymbol{x}_{i-1}+\boldsymbol{x}_{i}$ for all $i$, where we consider subscripts modulo 6. See Fig. 11. Then we have the following:

1. For any $\boldsymbol{x}$ such that $\boldsymbol{o x} \geq 1$ we have $\boldsymbol{x} \notin$ int $\operatorname{conv}\left\{\boldsymbol{x}_{0}, \boldsymbol{x}_{1}, \ldots, \boldsymbol{x}_{5}\right\}$.

2. For any unit vector $\boldsymbol{x}$ we have $\boldsymbol{x} \in \operatorname{conv}\left\{\boldsymbol{x}_{i-1}, \boldsymbol{x}_{i}, \boldsymbol{y}_{i}\right\}$ for some i, i.e.,

$$
\text { bd } C \subseteq \bigcup_{i \in \mathbf{Z}_{6}} \operatorname{conv}\left\{\boldsymbol{x}_{i-1}, \boldsymbol{x}_{i}, \boldsymbol{y}_{i}\right\}
$$

If $C$ is not a paralleloid, this can be strengthened to

$$
\text { bd } C \subseteq \bigcup_{i \in \mathbf{Z}_{6}} \operatorname{conv}\left\{\boldsymbol{x}_{i-1}, \boldsymbol{x}_{i}\right\} \cup \operatorname{int} \bigcup_{i \in \mathbf{Z}_{6}} \operatorname{conv}\left\{\boldsymbol{x}_{i-1}, \boldsymbol{x}_{i}, \boldsymbol{y}_{i}\right\}
$$

Lemma 14. Let the unit ball $C$ of a normed plane be nonparalleloid. Then there exists $\varepsilon>0$ depending only on $C$ such that the following holds. Given the minimum distance graph in Fig. 12, i.e., $\triangle \boldsymbol{p} \boldsymbol{p}_{+} \boldsymbol{q}$ and $\triangle \boldsymbol{q s}$ are equilateral with side length 1 , also $\boldsymbol{p}_{+} \boldsymbol{q}_{+}=\boldsymbol{q}_{+} \boldsymbol{r}_{+}=1$, with all other distances greater than or equal to $1 ; \boldsymbol{q}$ and $\boldsymbol{q}_{+}$are on the same side of $\overleftrightarrow{\boldsymbol{p p}_{+}}$, and $\boldsymbol{p}, \boldsymbol{p}_{+}, \boldsymbol{q}_{+}, \boldsymbol{s}, \boldsymbol{r}$ are in the displayed order around $\boldsymbol{q}$. Let 


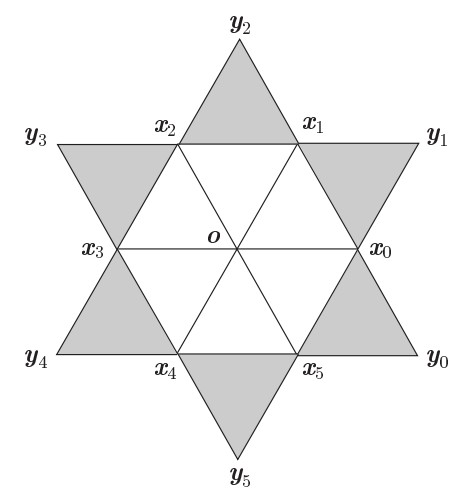

Fig. 11. Locating the unit ball in an equilateral triangular lattice.

$\boldsymbol{r}^{\prime}=\boldsymbol{r}+\left(\boldsymbol{p}_{+}-\boldsymbol{p}\right)$. Then $\boldsymbol{r}_{+}$and $\overleftrightarrow{\boldsymbol{p} \boldsymbol{r}}$ are on opposite sides of $\overleftrightarrow{\boldsymbol{p}_{+} \boldsymbol{r}^{\prime}}$, i.e., the angle from $\overrightarrow{\boldsymbol{p}_{+} \boldsymbol{r}^{\prime}}$ to $\overrightarrow{\boldsymbol{p}_{+} \boldsymbol{r}_{+}}$is negative (clockwise), and, furthermore,

$$
\left\|\frac{\boldsymbol{r}-\boldsymbol{p}}{\|\boldsymbol{r}-\boldsymbol{p}\|}-\frac{\boldsymbol{r}_{+}-\boldsymbol{p}_{+}}{\left\|\boldsymbol{r}_{+}-\boldsymbol{p}_{+}\right\|}\right\| \geq \varepsilon .
$$

Proof. We assume that

$$
\text { either } \overleftrightarrow{\boldsymbol{p r}} \| \overleftrightarrow{\boldsymbol{p}_{+} \boldsymbol{r}_{+}} \text {, or } \boldsymbol{r}_{+} \text {and } \overleftrightarrow{\boldsymbol{p r}} \text { are on the same side of } \overleftrightarrow{\boldsymbol{p}_{+} \boldsymbol{r}^{\prime}}
$$

and aim for a contradiction.

Without loss of generality we let $\boldsymbol{q}$ be the origin $\boldsymbol{o}$ and consider the triangular lattice generated by $\boldsymbol{p}$ and $\boldsymbol{p}_{+}$. We now locate the remaining points $\boldsymbol{r}, \boldsymbol{s}, \boldsymbol{q}_{+}, \boldsymbol{r}_{+}$with respect to this lattice. See Fig. 13.

Since

- $\boldsymbol{q}_{+}$is on the boundary of the unit ball $C+\boldsymbol{p}_{+}$,

- $\boldsymbol{o}$ and $\boldsymbol{q}_{+}$are on the same side of $\overleftrightarrow{\boldsymbol{p p}_{+}}$

- $\boldsymbol{q}_{+}$is inside $\varangle \boldsymbol{p}_{+} \boldsymbol{o s}$,

- and $\boldsymbol{q}_{+}$is not in the interior of the unit ball (since $\boldsymbol{o q _ { + }} \geq 1$ ),

it follows from Lemma 13 that

$$
\boldsymbol{q}_{+} \in \operatorname{conv}\left\{\boldsymbol{p}_{+}-\boldsymbol{p}, 2 \boldsymbol{p}_{+}-\boldsymbol{p}, 2 \boldsymbol{p}_{+}-2 \boldsymbol{p}\right\} .
$$

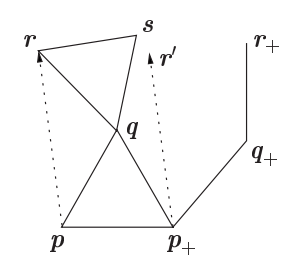

Fig. 12. Local analysis of nonconcave arc in Lemma 14. 


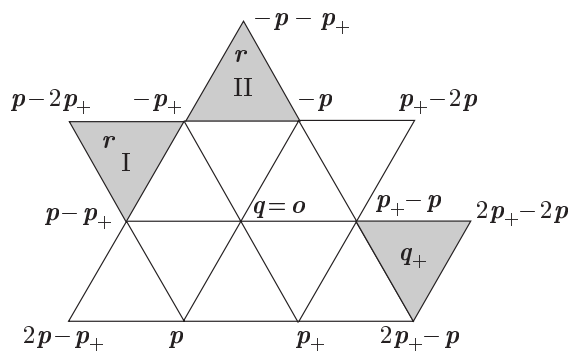

Fig. 13. Proof of Lemma 14

Applying Lemma 13 again to $\boldsymbol{p r} \geq 1=\boldsymbol{o r}$ we obtain that

$$
\boldsymbol{r} \notin \operatorname{conv}\left\{\boldsymbol{p}, \boldsymbol{p}-\boldsymbol{p}_{+}, 2 \boldsymbol{p}-\boldsymbol{p}_{+}\right\} \backslash\left\{\boldsymbol{p}-\boldsymbol{p}_{+}\right\} .
$$

Similarly, applying Lemma 13 to $\boldsymbol{p}_{+} \boldsymbol{s} \geq 1=\boldsymbol{o s}$ we obtain that

$$
\boldsymbol{s} \notin \operatorname{conv}\left\{\boldsymbol{p}_{+}, \boldsymbol{p}_{+}-\boldsymbol{p}, 2 \boldsymbol{p}_{+}-\boldsymbol{p}\right\} \backslash\left\{\boldsymbol{p}_{+}-\boldsymbol{p}\right\} .
$$

Also, if $\boldsymbol{r} \in \operatorname{conv}\left\{-\boldsymbol{p}, \boldsymbol{p}_{+}-\boldsymbol{p}, \boldsymbol{p}_{+}-2 \boldsymbol{p}\right\} \backslash\{-\boldsymbol{p}\}$, then, since $\boldsymbol{s}$ is in the interior of $\varangle \boldsymbol{p}_{+} \boldsymbol{o r}$ and $\boldsymbol{o s}=1$, we must also have $\boldsymbol{s} \in \operatorname{conv}\left\{-\boldsymbol{p}, \boldsymbol{p}_{+}-\boldsymbol{p}, \boldsymbol{p}_{+}-2 \boldsymbol{p}\right\} \backslash\{-\boldsymbol{p}\}$. Then by Lemma 12 we obtain $\boldsymbol{s}=\boldsymbol{p}_{+}-\boldsymbol{p}$ and $\boldsymbol{r}=-\boldsymbol{p}$, contradicting the assumption on $\boldsymbol{r}$. Thus there are two cases to consider:

I. $\boldsymbol{r} \in \operatorname{conv}\left\{\boldsymbol{p}-\boldsymbol{p}_{+},-\boldsymbol{p}_{+}, \boldsymbol{p}-2 \boldsymbol{p}_{+}\right\}$,

II. $\boldsymbol{r} \in \operatorname{conv}\left\{-\boldsymbol{p},-\boldsymbol{p}_{+},-\boldsymbol{p}-\boldsymbol{p}_{+}\right\}$.

Case I. In this case $\boldsymbol{s} \in \operatorname{conv}\left\{-\boldsymbol{p},-\boldsymbol{p}_{+},-\boldsymbol{p}-\boldsymbol{p}_{+}\right\}$by Lemma 12. It follows from assumption (12) that $\boldsymbol{o}$ and $\boldsymbol{r}_{+}$cannot be on opposite sides of the line through $\boldsymbol{p}_{+}$and $-\boldsymbol{p}$. Applying Lemma 13 to $\boldsymbol{q}_{+} \boldsymbol{r}_{+}=1$ with all possible positions of $\boldsymbol{q}_{+}$, and also to $\boldsymbol{o} \boldsymbol{r}_{+}, \boldsymbol{p} \boldsymbol{r}_{+}, \boldsymbol{p}_{+} \boldsymbol{r}_{+} \geq 1$, we obtain that $\boldsymbol{r}_{+}=-\boldsymbol{p}$ is the only possibility. Since $\boldsymbol{q}_{+} \boldsymbol{r}_{+}=1$, by Lemma 13 we must have $\boldsymbol{q}_{+}=\boldsymbol{p}_{+}-\boldsymbol{p}$. Also, since $\boldsymbol{r}_{+} \boldsymbol{s} \geq 1$, we must have $\boldsymbol{s}=-\boldsymbol{p}_{+}$, and since $\boldsymbol{r s} \geq 1$, we must have $\boldsymbol{r}=\boldsymbol{p}-\boldsymbol{p}_{+}$. Then (12) is contradicted.

Case II. In this case $\boldsymbol{s} \in \operatorname{conv}\left\{-\boldsymbol{p}, \boldsymbol{p}_{+}-\boldsymbol{p}, \boldsymbol{p}_{+}-2 \boldsymbol{p}\right\}$ by Lemma 12. Let $\boldsymbol{s}_{1}=\boldsymbol{s}+\boldsymbol{p}_{+}, \boldsymbol{s}_{2}=$ $\boldsymbol{s}+p_{+}-p, s_{3}=s-p, s_{4}=s-p_{+}, s_{5}=s+p-p_{+}, s=s+p$. Let $s^{\prime}$ be the intersection of the line through $\boldsymbol{p}_{+}-\boldsymbol{p}$ and $\boldsymbol{s}$, and the line though $-\boldsymbol{p}$ and $\boldsymbol{p}_{+}-2 \boldsymbol{p}$. Let $\boldsymbol{s}^{\prime \prime}$ be the intersection of the line through $-\boldsymbol{p}$ and $\boldsymbol{s}$, and the line through $\boldsymbol{p}_{+}-\boldsymbol{p}$ and $\boldsymbol{p}_{+}-2 \boldsymbol{p}$. Let $s_{1}^{\prime}=\boldsymbol{s}^{\prime}+\boldsymbol{p}_{+}$and $\boldsymbol{s}_{1}^{\prime \prime}=\boldsymbol{s}^{\prime \prime}+\boldsymbol{p}_{+}$. Let $\boldsymbol{a}$ be the intersection of the line through $-\boldsymbol{p}$ and $\boldsymbol{p}_{+}-\boldsymbol{p}$, and the segment $\boldsymbol{s}_{4} \boldsymbol{s}_{5}, \boldsymbol{a}^{\prime}=\boldsymbol{a}+\boldsymbol{p}_{+}-\boldsymbol{p}$, and let $\boldsymbol{b}$ be the intersection of the line through $-\boldsymbol{p}$ and $\boldsymbol{p}_{+}-\boldsymbol{p}$, and the segment $\boldsymbol{s}_{1} \boldsymbol{s}_{6}$. See Fig. 14.

Since $\boldsymbol{s} \boldsymbol{r}=1$, by Lemma $13 \boldsymbol{r}$ cannot be in the interior of hexagon $\boldsymbol{s}_{1} \boldsymbol{s}_{2} \cdots s_{6}$. Thus $\boldsymbol{r}$ and $\boldsymbol{o}$ are not on the same side of line $\overleftrightarrow{\boldsymbol{a p}}$. By (12) it follows that $\boldsymbol{r}_{+}$and $\boldsymbol{o}$ are not on opposite sides of line $\overleftrightarrow{\boldsymbol{a}_{\boldsymbol{p}}}$.

Since $-\boldsymbol{p}, \boldsymbol{p}_{+}-\boldsymbol{p}$ and $\boldsymbol{s}$ are all unit vectors, there is no unit vector in the interior of $\varangle \boldsymbol{s}^{\prime \prime} \boldsymbol{s} \boldsymbol{s}^{\prime}$, by convexity of the unit ball. Translating by $\boldsymbol{p}_{+}$we find that since $\boldsymbol{p}_{+} \boldsymbol{q}_{+}=1, \boldsymbol{q}_{+}$ is not in the interior of $\varangle s_{1}^{\prime \prime} s_{1} s_{1}^{\prime}$. 


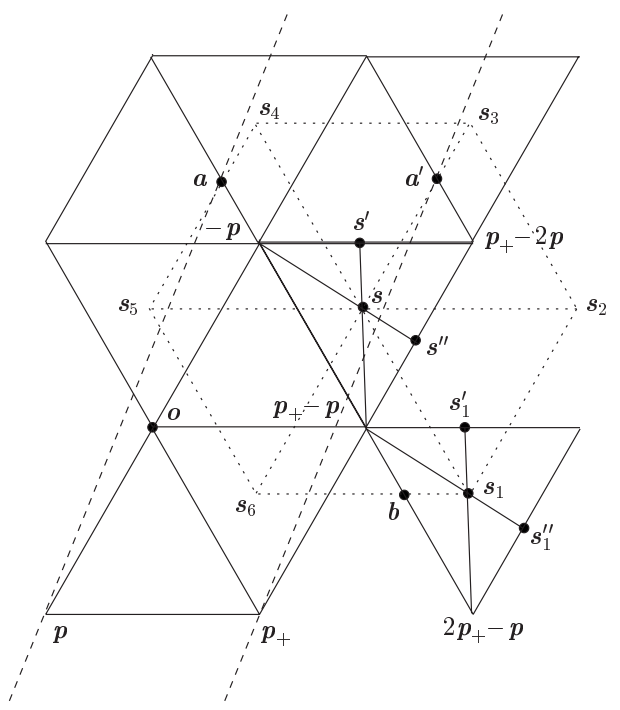

Fig. 14. Case II in the proof of Lemma 14.

Since $s q_{+} \geq 1, q_{+}$cannot be in the interior of hexagon $s_{1} \cdots s_{6}$. It follows that

$$
\boldsymbol{q}_{+} \in \operatorname{conv}\left\{\boldsymbol{b}, \boldsymbol{s}_{1}, \boldsymbol{s}_{1}^{\prime \prime}, 2 \boldsymbol{p}_{+}-\boldsymbol{p}\right\}
$$

By applying Lemma 13 to $\boldsymbol{q}_{+} \boldsymbol{r}_{+}=1$ with all possible positions of $\boldsymbol{q}_{+}$(noting that $\boldsymbol{q}_{+} \neq$ $2 \boldsymbol{p}_{+}-\boldsymbol{p}$ since $\boldsymbol{q}_{+}$cannot be on $\overleftrightarrow{\boldsymbol{p}_{+}}$by the lemma's hypothesis), we obtain that the possible positions for $\boldsymbol{r}_{+}$are all either in the interior of the hexagon $s_{1} \cdots s_{6}$ or the interiors of the analogous hexagons with centres $\boldsymbol{o}, \boldsymbol{p}$ or $\boldsymbol{p}_{+}$. However, since $\boldsymbol{r}_{+} \boldsymbol{s}, \boldsymbol{r}_{+} \boldsymbol{o}, \boldsymbol{r}_{+} \boldsymbol{p}, \boldsymbol{r}_{+} \boldsymbol{p}_{+} \geq 1$, by Lemma $13, \boldsymbol{r}_{+}$cannot be in any of these regions, a contradiction.

Thus we have shown that (12) is false.

If an $\varepsilon>0$ cannot be found as required, then for each $n \geq 1$ we can find a minimum distance graph as described in the hypothesis of the lemma, but for which

$$
\left\|\frac{\boldsymbol{r}-\boldsymbol{p}}{\|\boldsymbol{r}-\boldsymbol{p}\|}-\frac{\boldsymbol{r}_{+}-\boldsymbol{p}_{+}}{\left\|\boldsymbol{r}_{+}-\boldsymbol{p}_{+}\right\|}\right\|<\frac{1}{n} .
$$

Taking a convergent subsequence, we obtain a minimum distance graph still satisfying the hypothesis of the lemma, but now $\overleftrightarrow{\boldsymbol{r p}} \| \overleftrightarrow{\boldsymbol{r}_{+} \boldsymbol{p}_{+}}$, which we have already shown to be false.

By Theorem 4 we only have to show the following to finish the proof of Theorem 2:

Lemma 15. Let $M(C)$ be a normed plane with $C$ not a paralleloid. Then for sufficiently large $m$ depending only on $M(C)$ we have $\boldsymbol{s}_{i}=\boldsymbol{r}_{i+1}(i=a, a+1$ and $a+2)$ for some $1 \leq a \leq 2 m-11$. 
Proof. Note that we can apply Lemma 14, since it follows from $\tau_{1}+\cdots+\tau_{2 m-3}<\pi / 3$ that $\boldsymbol{q}_{i}$ and $\boldsymbol{q}_{i+1}$ are on the same side of $\overleftrightarrow{\boldsymbol{p}_{i} \boldsymbol{p}_{i+1}}$. We take $\varepsilon>0$ as in Lemma 14, and then $\delta>0$ as in Lemma 11 applied to $\varepsilon / 3$. Let $m>4+2 / \delta+41 / \delta \varepsilon$ (to be on the safe side). Let $\boldsymbol{u}_{i}=\left(\boldsymbol{r}_{i}-\boldsymbol{p}_{i}\right) /\left\|\boldsymbol{r}_{i}-\boldsymbol{p}_{i}\right\|$ for each $i=1, \ldots, 2 m-5$. Suppose that for any $a=1, \ldots, 2 m-11$ we have $s_{i} \neq \boldsymbol{r}_{i+1}$ for at least one $i=a, a+1, a+2$. Since for each $i, \tau_{i+1} \geq 2 \pi / 3-\mu\left(\varangle \boldsymbol{p}_{i+1} \boldsymbol{q}_{i} \boldsymbol{s}_{i}\right)$ (by Lemma 4), it follows that for a total of at most $\pi / 3 \delta i$ 's we have $\mu\left(\varangle \boldsymbol{p}_{i+1} \boldsymbol{q}_{i} \boldsymbol{s}_{i}\right) \leq 2 \pi / 3-\delta$. Similarly, for a total of at most $\pi / 3 \delta i$ 's we have $\mu\left(\varangle \boldsymbol{r}_{i} \boldsymbol{q}_{i} \boldsymbol{p}_{i}\right) \leq 2 \pi / 3-\delta$. By Lemma 11, the choice of $m$ and the pigeon-hole principle, there is a stretch of at least $39 / \varepsilon$ consecutive $i$ 's, say $i=1, \ldots,\lceil 39 / \varepsilon\rceil$, such that $\left\|\boldsymbol{u}_{i+1}-\boldsymbol{u}_{i}\right\|<\varepsilon / 3$ whenever $\boldsymbol{s}_{i}=\boldsymbol{r}_{i+1}$. Also, by Lemma 14, $\left\|\boldsymbol{u}_{i+1}-\boldsymbol{u}_{i}\right\| \geq \varepsilon$ whenever $\boldsymbol{s}_{i} \neq \boldsymbol{r}_{i+1}$. By the triangle inequality, $\left\|\boldsymbol{u}_{3(i+1)}-\boldsymbol{u}_{3 i}\right\| \geq \varepsilon / 3$, and the sequence $\boldsymbol{u}_{3 i}$ is in order on the boundary of the unit ball. Thus the length (in the norm) of the arc on the boundary of the unit ball from $\boldsymbol{u}_{0}$ to $\boldsymbol{u}_{3\lceil 13 / \varepsilon\rceil}$ is at least $\lceil 13 / \varepsilon\rceil \varepsilon / 3>4$. It follows that the circumference of the boundary of the unit ball is greater than 8 , a contradiction (see [8]).

\section{Acknowledgment}

The author thanks the referees for valuable remarks and suggestions resulting in a substantial improvement over a previous version of this paper.

\section{References}

1. P. Brass, Erdős distance problems in normed spaces, Comput. Geom. 6 (1996), 195-214.

2. G. Csizmadia, On the independence number of minimum distance graphs, Discrete Comput. Geom. 20 (1998), 179-187.

3. P. Erdős, Some combinatorial and metric problems in geometry, in Intuitive Geometry (K. Böröczky and G. Fejes Tóth, eds.), Colloquia Mathematica Societatis János Bolyai, vol. 48, North-Holland, Amsterdam, 1987, pp. 167-177.

4. B. Grünbaum, On a conjecture of H. Hadwiger, Pacific J. Math. 11 (1961), 215-219.

5. J. Pach and G. Tóth, On the independence number of coin graphs, Geombinatorics 6 (1996), 30-33.

6. R. Pollack, Increasing the minimum distance of a set of points, J. Combin. Theory Ser. A 40 (1985), 450.

7. K. J. Swanepoel, Gaps in convex disc packings with an application to 1-Steiner minimum trees, Monatsh. Math. 129 (2000), 217-226.

8. A. C. Thompson, Minkowski Geometry, Encyclopedia of Mathematics and its Applications 63, Cambridge University Press, Cambridge, 1996. 